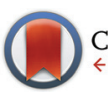

CrossMark

\&lick for updates

Cite this: Dalton Trans., 2014, 43

14181

Received 16th April 2014,

Accepted 3rd June 2014

DOI: 10.1039/c4dt01130a

www.rsc.org/dalton

\title{
New avenues in the directed deprotometallation of aromatics: recent advances in directed cupration
}

\author{
Philip J. Harford, ${ }^{a}$ Andrew J. Peel, ${ }^{a}$ Floris Chevallier, ${ }^{\text {b }}$ Ryo Takita,,${ }^{c, d}$ \\ Florence Mongin, ${ }^{* b}$ Masanobu Uchiyama*c,d and Andrew E. H. Wheatley*a
}

\begin{abstract}
Recent advances in the selective deprotometallation of aromatic reagents using alkali metal cuprates are reported. The ability of these synergic bases to effect deprotonation under the influence of a directing group is explored in the context of achieving new and more efficient organic transformations whilst encouraging greater ancillary group tolerance by the base. Developments in our understanding of the structural chemistry of alkali metal cuprates are reported, with both Gilman cuprates of the type $\mathrm{R}_{2} \mathrm{CuLi}$ and Lipshutz and related cuprates of the type $\mathrm{R}_{2} \mathrm{Cu}(\mathrm{X}) \mathrm{Li}_{2}(X=$ inorganic anion) elucidated and rationalised in terms of ligand sterics. The generation of new types of cuprate motif are introduced through the development of adducts between different classes of cuprate. The use of DFT methods to interrogate the mechanistic pathways towards deprotonative metallation is described. Theoretical modelling of in situ rearrangements undergone by the cuprate base are discussed, with a view to understanding the relationship between $R_{2} C u L i$ and $R_{2} C u(X) L_{2}$, their interconversion and the implications of this for cuprate reactivity. The advent of a new class of adduct between different cuprate types is developed and interpreted in terms of the options for expelling $\mathrm{LiX}$ from $\mathrm{R}_{2} \mathrm{Cu}(\mathrm{X}) \mathrm{Li}_{2}$. Applications in the field of medicinal chemistry and (hetero)arene derivatization are explored.
\end{abstract}

\section{General introduction}

Deprotolithiation has proved to be an enormously powerful method by which to effect the regioselective functionalization of aromatics. ${ }^{1}$ Historically, bases such as organolithiums and lithium dialkylamides have typically been employed for this purpose. However, either because they are highly polar or

\footnotetext{
${ }^{a}$ Department of Chemistry, University of Cambridge, Lensfield Road, Cambridge, CB2 1EW, UK. E-mail: aehw2@cam.ac.uk; Fax:+44 (0)1223 336362; Tel: $+44(0) 1223763966$

${ }^{b}$ UMR 6226, Institut des Sciences Chimiques de Rennes, Chimie et Photonique Moléculaires, Université de Rennes 1-CNRS, Campus de Beaulieu, Bâtiment 10A, Case 1003, 35042 - Rennes Cedex, France.E-mail: Florence.Mongin@univ-rennes1.fr ${ }^{c}$ Advanced Elements Chemistry Research Team, RIKEN Center for Sustainable Resource Science (CSRS), and Elements Chemistry Laboratory, RIKEN, 2-1 Hirosawa, Wako-shi, Saitama 351-0198, Japan. E-mail: uchiyama@mol.f.u-tokyo.ac.jp ${ }^{d}$ Graduate School of Pharmaceutical Sciences, The University of Tokyo, 7-3-1 Hongo, Bunkyo-ku, Tokyo 113-0033, Japan
}

because they generate polar aryllithiums, their use for the functionalization of sensitive substrates (e.g. bearing functional groups that are prone to nucleophilic attack, rearrangement or elimination) requires strictly controlled conditions. In addition, organolithium compounds react with electrophiles, such as aroyl chlorides, through double addition, limiting synthetic possibilities by rendering the direct synthesis of ketones unlikely.

Recently, the use of organic bases containing two metals has come to the fore. These so-called synergic reagents exhibit behaviour that is different from that of the corresponding monometal bases. Whilst the field is a challenging one, since its inception ${ }^{2}$ in 1999 with the development of the putative lithium zincate $\mathrm{Bu}_{2}^{t} \mathrm{Zn}(\mathrm{TMP}) \mathrm{Li}$ (TMP $=2,2,6,6$-tetramethylpiperidide), a variety of complexes of the type $(\mathrm{R})_{m}\left(\mathrm{R}^{\prime}\right)_{n} \mathrm{MM}^{\prime}(\mathrm{M}=$ alkali metal, $\mathbf{M}^{\prime}=$ lower polarity metal; $\mathrm{R}, \mathrm{R}^{\prime}=$ alkyl, amido, chloro, etc.), and in particular those containing the very hindered TMP ligand, have been reported to act as efficient and
Philip Harford obtained an MSc degree in Natural Sciences in 2008 from the University of Cambridge and is currently studying for his PhD there under the supervision of Dr Andrew Wheatley. His research is focussing on the synthesis of heterobimetallic lithium cuprate bases with particular interest in their structures as derived from X-ray crystallographic studies.
Andrew Peel completed his degree in Natural Sciences at the University of Cambridge in 2013. He is currently undertaking a PhD in Chemistry in the Wheatley group. His research presently focuses on lithium amidocuprate chemistry, with his principal interests being in chemical crystallography. His other interests include growing exotic chilli peppers and playing guitar. 
selective superbases. ${ }^{3}$ These complexes - which now feature $\mathrm{Zn}, \mathrm{Al}, \mathrm{Cu}, \mathrm{Mn}$ and $\mathrm{Mg}$ as the lower polarity metal - all exhibit 'ate formulations, and, as such, reveal both anionic activation ${ }^{4}$ and reduced aggregation ${ }^{5}$ when suitable reaction media are employed. Recently, more elaborate versions of these complexes have appeared; these include salt-activated organometallic compounds (e.g. "turbo"-Grignard reagents), and synergic pairs of metal amides that complement each other in deprotometallation reactions. ${ }^{3 d, g}$

The present Perspective depicts the behaviour towards aromatics of lithium cuprates specifically, and the development of the concept of Directed ortho Cupration (DoC) that was first reported in 2007. ${ }^{6}$ Though they were first prepared in the 1950 s, evidence for the actual nature of lithium cuprates has been slow to be forthcoming and it is only recently that solidstate structural data has appeared for examples of these complexes designed to behave as deprotonating agents. The present article is divided into five main parts. After this introduction, cuprate synthesis and structural characterisation are detailed in the second part. Understanding of the structural principles at work in lithium cuprates is important in order to fully appreciate differences in their reactivities. As is discussed below, there has been much debate into whether such differences in reactivity can be explained in terms of reagent structure. Recently, however, very interesting work has gone a long way to explaining the contrasting activities of cuprates (e.g. 7 and 12, see below) that are now known to be structurally analogous. ${ }^{7}$ After this structural discussion, the scope and applications of aromatic directed cupration are presented. Discussion then shifts to the theoretical interrogation of these systems with a view to understanding their mechanistic chemistry. Finally, the concluding section seeks to draw together structural evidence and theoretical work with a view to rationalising the reported reactions and identifying future directions.

\section{Advances in the solid-state structural chemistry of alkali metal amidocuprates}

\subsection{Lipshutz cuprates: the solid-state evidence}

The most investigated method by which organocopper compounds can provide reactive organic anions is via organo- cuprates. First reported in 1952, Gilman noted that the addition of 1 equiv. MeLi to CuI resulted in the formation of a highly reactive and ether-insoluble yellow product thought to be $(\mathrm{MeCu})_{\infty}$. This dissolved upon addition of further $\mathrm{MeLi}^{8}$ to give (empirically) the lithium cuprate $\mathrm{Me}_{2} \mathrm{CuLi} \mathrm{1.}{ }^{9}$ In 1981, the replacement of $\mathrm{CuI}$ with $\mathrm{CuCN}$ to give more reactive cuprates for halide substitution reactions was proposed. At this time it was argued that the cyanide anion $\sigma$-bonded to $\mathrm{Cu},{ }^{10}$ though while some NMR studies were interpreted in terms of the presence of such a motif in solution, ${ }^{11}$ solid-state evidence for the exact nature of so-called 'Lipshutz cuprates' remained elusive.

Initial crystallographic evidence for the structures of lithium cuprates appeared only in the early 1980s, with publication of the structures of $\left[\mathrm{Cu}_{5} \mathrm{Ph}_{6}\right]^{-}[\mathrm{Li} \cdot 4 \mathrm{THF}]^{+} 2$ and $\left[\mathrm{Li}_{2} \mathrm{Cu}_{3} \mathrm{Ph}_{6}\right]_{2}\left[\mathrm{Li}_{4} \mathrm{Cl}_{2} \cdot 10 \mathrm{Et}_{2} \mathrm{O}\right] 3 .^{12,13}$ Shortly thereafter, van Koten revealed the solid-state structure of the dimer of $\left(\mathrm{C}_{6} \mathrm{H}_{4}\left\{\mathrm{CH}_{2} \mathrm{NMe}_{2}\right\}-2\right)_{2} \mathrm{CuLi}$ 4. $^{14}$ This structure, in which an 8membered metallocycle was supported by amine chelation of the alkali metal centres agreed well with that previously proposed based on a combination of multinuclear and variabletemperature NMR spectroscopic studies. ${ }^{15}$ This period also saw the publication of evidence suggestive of the true nature of Lipshutz cuprates in particular. Attempts to fabricate lithium cuprates from CuI and (halide-free) organolithiums in the presence of a crown ether yielded both $\left[\mathrm{CuMe}_{2}\right]^{-}$and $\left[\mathrm{CuPh}_{2}\right]^{-}$ions. ${ }^{16}$ These linear cuprate ions have been defined as so-called 'lower-order', with the possibility of 'higher-order' cuprate formation, in which copper is 3-coordinate, having been previously invoked to explain the enhanced reactivity of Lipshutz species. ${ }^{10,17}$ By 1990 the lack of data supporting such higher-order cuprates prompted the publication of NMR spectroscopic studies on mixtures of the bis(organyl)cuprates $\mathrm{R}_{2}$ CuLi with $\mathrm{LiX}(\mathrm{R}=\mathrm{Me}, \mathrm{Ph} ; \mathrm{X}=\mathrm{I}, \mathrm{CN})$. The identical nature of the resulting data was argued to be consistent with lowerorder structures in solution. ${ }^{18}$ However, the controversy continued with the presentation of contrary spectroscopic evidence, with the chemical shifts of the $\mathrm{Me}_{2} \mathrm{CuLi} / \mathrm{LiI}$ system changing upon the addition of LiCN and the postulated formation of a $\mathrm{Cu}-\mathrm{CN}$ interaction. ${ }^{19}$ That said, alternative explanations, e.g. LiCN coordination to an $\mathrm{R}$ group, remained, and this ambiguity led to the theoretical investigation by Snyder of possible structural candidates (see section 4). This led to the conclusion that higher-order structures were less stable than their lowerorder analogues. ${ }^{20}$ Whilst in response to these finding it was
Floris Chevallier is an Associate Professor at the University of Rennes 1 (Rennes, France). He studied (organic) chemistry at the universities of Orléans and Nantes. He received his PhD in 2004 under the supervision of Prof. Jean-Paul Quintard, for whom he worked on organotin chemistry. In 2005, he joined the group of Prof. Bernhard Breit at the Albert-Ludwigs-Universität Freiburg (Freiburg, Germany) as a postdoctoral researcher, where he focused on supramolecular catalysis. His current research interests include organometallic methodologies and molecular self-assemblies.
Ryo Takita obtained his PhD in 2006 from the University of Tokyo with Prof. Masakatsu Shibasaki. From 2006 to 2007, he worked as a postdoctoral Fellow at Massachusetts Institute of Technology with Professor Timothy M. Swager. He became an Assistant Professor at Kyoto University (2007-2010) and University of Tokyo (2010-2012), and was promoted to Lecturer (2012-2014). He is currently a Deputy Team Leader at RIKEN Center for Sustainable Resource Science (CSRS). His research interests include organic chemistry for unexplored reactivity and functions. 
proposed that $\mathrm{CN}^{-}$might $\sigma$-bond to lithium and $\pi$-bond to copper ${ }^{21}$ further theoretical work suggested the former coordinative mode to be more likely than the latter. ${ }^{22}$

It is only since 1998 that unambiguous structural evidence for the behaviour of cyanide ligands in the presence of $\mathrm{Cu}(\mathrm{I})$ organometallics has appeared in the literature. Initial reports verified the solid-state structures of ion-separated $\left[\mathrm{Bu}_{2}^{t} \mathrm{Cu}\right]^{-}$$\left[(\mu \text {-CN)(Li·THF-PMDTA })_{2}\right]^{+} 5$ (PMDTA $=N, N, N^{\prime}, N^{\prime \prime}, N^{\prime \prime}$-pentamethyldiethylenetriamine) and the polymer of closely related $\left.\left\{\left(2-\mathrm{Me}_{2} \mathrm{NCH}_{2} \mathrm{C}_{6} \mathrm{H}_{4}\right)_{2} \mathrm{Cu}\right\}(\mu-\mathrm{CN})(\mathrm{Li} \cdot 2 \mathrm{THF})_{2}\right\} 6$ (Fig. 1). ${ }^{23,24}$ In the first of these examples, the use of a strong Lewis base (PMDTA) has induced ion-separation by extruding $\mathrm{Li}^{+}$from the cuprate moiety. Concordant with this and, as is described in section 4, based both on crystallographic and theoretical studies, the ability of the cuprate component of the structure to interact with an alkali metal is contingent not only on the Lewis basicity of the solvent but also on the nature of the $\mathrm{Cu}$-bonded organyl groups. In the case of $\mathbf{5}$, the adoption of a $\mathrm{Cu}\left(\mu-\mathrm{Bu}^{t}\right) \mathrm{Li}$ motif is not strongly favoured by the choice of potential bridging group. Accordingly, therefore, separated and linear $\left[\mathrm{Bu}_{2}^{t} \mathrm{Cu}\right]^{-}$and $\left[(\mu-\mathrm{CN}) \mathrm{Li}_{2}\right]^{+}$ions are noted. In contrast, the absence of PMDTA and the utilisation of bifunctional organyl groups in the cuprate ion instead lead to ion-association in $\mathbf{6}$ via the formation of $\mathrm{N}\left(\mathrm{Me}_{2}\right)$ - $\mathrm{Li}$ interactions.

The issue of cyanide incorporation by cuprates has only recently been revisited. This work first appeared in 2007, with attempts to fabricate and structurally elucidate the proposed Lipshutz cuprate afforded by treating TMPLi with 0.5 equiv. of CuCN (Scheme 1). The imperative for this investigation came from the first reports of Directed ortho Cupration (DoC) facilitated by the use of cuprate moieties that incorporate sterically demanding amide ligands. ${ }^{6}$ As noted in section 3.2, a reagent with the putative (Lipshutz) formulation (TMP) $)_{2} \mathrm{Cu}(\mathrm{CN}) \mathrm{Li}_{2}$ was found to offer significant potential for aromatic homocoupling processes. X-ray diffraction on the isolated crystals established
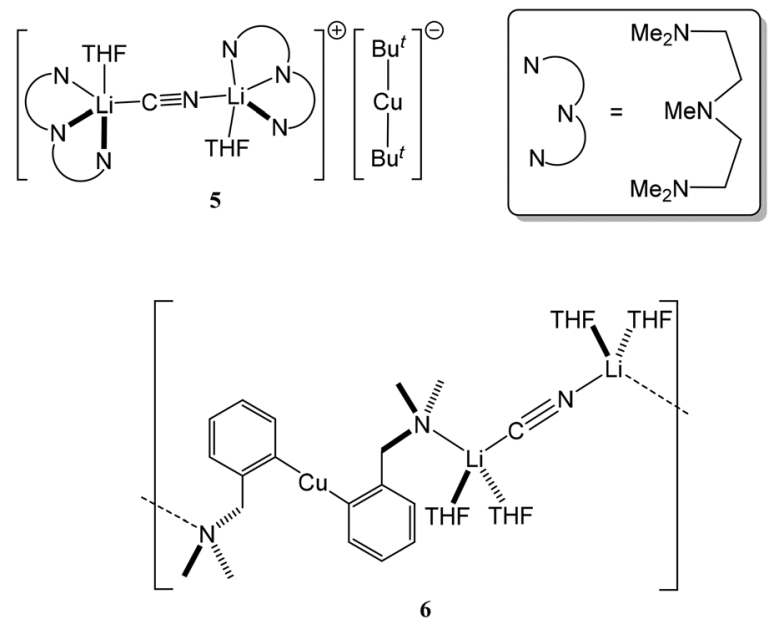

Fig. 1 The first solid-state evidence for lithium cyanocuprate structures was revealed by ion-separated 5 and the polymer of 6 .

the structure as being a dimer based on monomers of $(\mathrm{TMP})_{2} \mathrm{Cu}(\mathrm{CN}) \mathrm{Li}_{2} \cdot \mathrm{THF} 7$ (Fig. 2). The core is based on a classical $(\mathrm{NLi})_{2}$ cyclic architecture synonymous with the metallocycle formation of Snaith's vertical ${ }^{25}$ and lateral ${ }^{26}$ ring aggregation principles. Peripheral to this core are two cuprate components, based on approximately linear $\mathrm{N}-\mathrm{Cu}-\mathrm{N}$ motifs. The metal-bridging potential of the amido ligands is clearly demonstrated. Crucially, in terms of understanding the structural properties of cyanide-containing cuprates, the ion-associated species formed does not contain a bonded CuCN component in the solid-state. Instead, it closely approximates to the most stable of the structures computed by Snyder in $1994,{ }^{20}$ with the cyanide group bridging between alkali metal ions. The manner in which it does this is different for the various metals in the aggregated structure, with each cyanide interacting with three

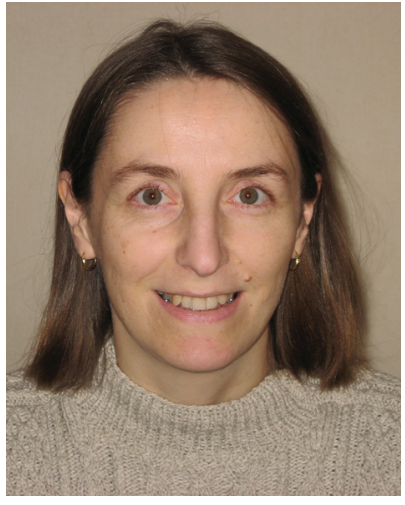

Florence Mongin
Florence Mongin obtained her PhD in Chemistry in 1994 from the University of Rouen (France) under the supervision of Prof. Guy Queguiner. After two years at the Institute of Organic Chemistry of Lausanne (Switzerland) as a postdoctoral Fellow with Prof. Manfred Schlosser, she returned to the University of Rouen as an Assistant Professor in 1997 (HDR in 2003). She took up her present position in 2005 as Professor at the University of Rennes (France) and was appointed Junior Member of the Institut Universitaire de France in 2009. Her interests include the functionalization of aromatic compounds including heterocycles with recourse to mixed lithium-metal bases.

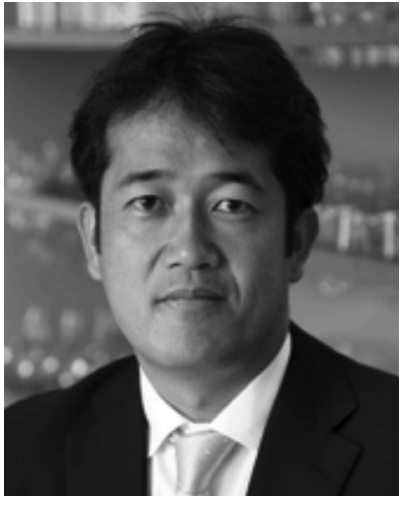

Masanobu Uchiyama
Masanobu Uchiyama obtained his PhD in 1998 from the University of Tokyo with Prof. Koichi Shudo. He worked as an Assistant Professor at Tohoku University (1995-2001) and University of Tokyo (2001-2003), and was promoted to lecturer (2003-2006). He became Associate Chief Scientist at RIKEN in 2006. He is currently a Full Professor at the University of Tokyo (from 2010) and Chief Scientist/Team Leader at RIKEN and RIKEN CSRS (from 2013). His research interests are in the area of synthetic organic chemistry with an emphasis on organometallic, physical, and computational chemistry. 

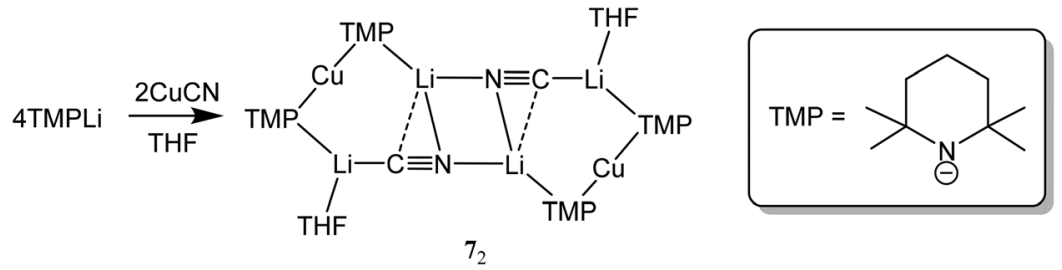

Scheme 1 Formation of the dimer of Lipshutz bis(amido)cuprate 7.

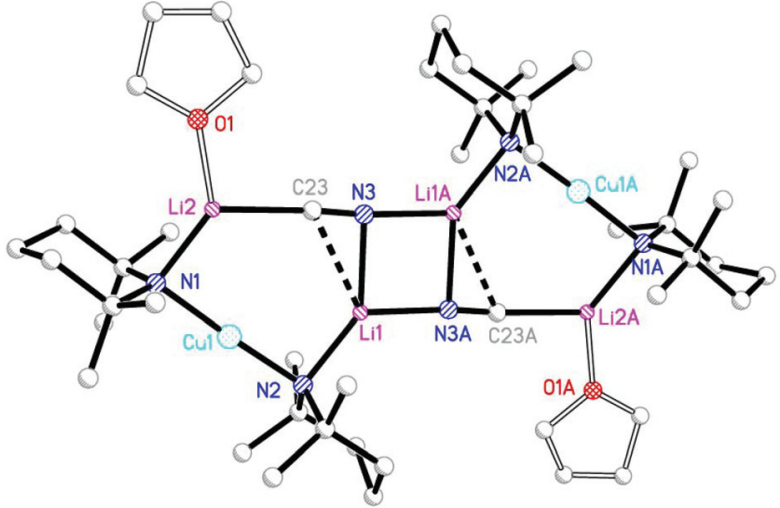

Fig. 2 Crystal structure ( $\mathrm{H}$ atoms omitted) of the dimer of Lipshutz cuprate 7.

different alkali metal centres; spanning two metal ions in near linear $\sigma$-fashion and exhibiting an offset side-on bond to the third.

\subsection{Extending the principle: Lipshutz-type structures}

The structural motif revealed by 7 turns out to have a great deal in common with the 6-membered metallocyclic lithio-

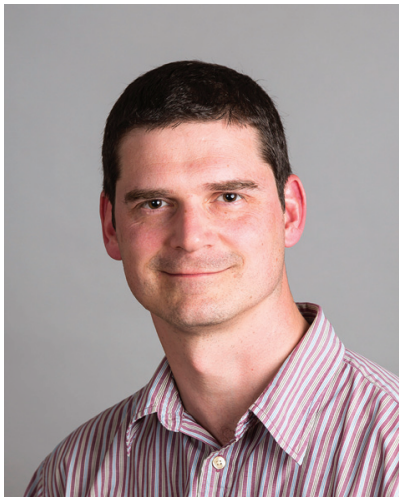

Andrew Wheatley
Andrew Wheatley obtained his BSc from the University of Kent at Canterbury in 1995. He then worked at Cambridge under the guidance of $\mathrm{Dr}$ Ron Snaith, receiving his $P h D$ in 1999 and an MA (Cantab) in 2002. After his PhD studies he undertook a Research Fellowship at Gonville \& Caius College before becoming a University Lecturer at Cambridge (2000) and a Fellow of Fitzwilliam College. He has received the Harrison Memorial Medal from the RSC and more recently he has been a Visiting Associate Professor at Tohoku University, Japan. He is now a University Senior Lecturer at Cambridge. cuprate $\left(\mathrm{Ph}_{2} \mathrm{~N}\right)_{2} \mathrm{Cu}\left(\mathrm{NPh}_{2}\right) \mathrm{Li}_{2} \cdot 2 \mathrm{OEt}_{2}$ 8. $^{27}$ This species revealed a solid-state monomer comprising a near linear geometry at $\mathrm{Cu}$ and both $\mathrm{Cu}-(\mu-\mathrm{N})-\mathrm{Li}$ and $\mathrm{Li}-(\mu-\mathrm{N})-\mathrm{Li}$ bridges (Fig. 3). Taken together, the observation of 7 and 8 suggests that there is nothing unique in the inter-lithium bridging activity of cyanide in the former complex. This idea led us to propose the creation of a series of what might be called 'Lipshutz-type' cuprates. These have the formulation $\mathrm{R}_{2} \mathrm{Cu}(\mathrm{X}) \mathrm{Li}_{2}$, such that for $7 \mathrm{X}$ $=\mathrm{CN}$. This idea has been tested by the replacement of CuCN with $\mathrm{CuHal}(\mathrm{Hal}=\mathrm{Cl}, \mathrm{Br}, \mathrm{I})$ in essentially the same synthetic sequence as that which yielded 7 (Scheme 2). In the first instance, $\mathrm{CuCl}$ was investigated (our interest in this deriving from its ability to promote the deprotonative metallation of 2chloropyridine as a precursor to benzoylation, see section 3.3). Initial work towards the isolation and characterisation of halide-containing reagent mixtures focused on the deployment of TMPLi and $\mathrm{CuCl}$, with data quickly establishing that solvent conditions capable of promoting the retention of in situformed LiCl in solution (where it is capable of combining with putative $\mathrm{R}_{2} \mathrm{CuLi}$ ) promoted cuprate reactivity. Importantly, attempts to replicate organic transformations using pre-<smiles>CCO[Al]1N(c2ccccc2)[Al](c2ccccc2)N(c2ccccc2)[Al](c2ccccc2)N1c1ccccc1</smiles>

Fig. 3 Monomeric structure of amidocuprate 8.

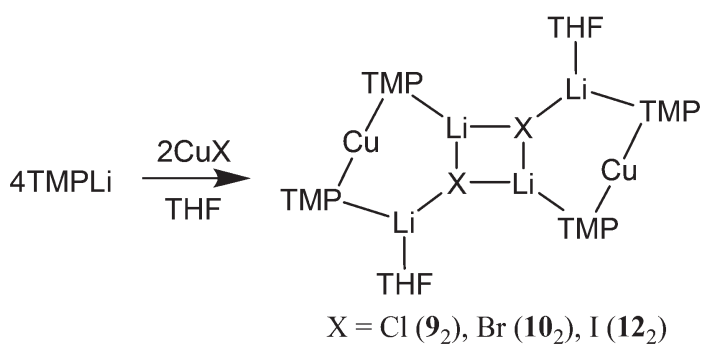

Scheme 2 Formation of the dimers of Lipshutz-type bis(amido)cuprates 9,10 and 12 . 


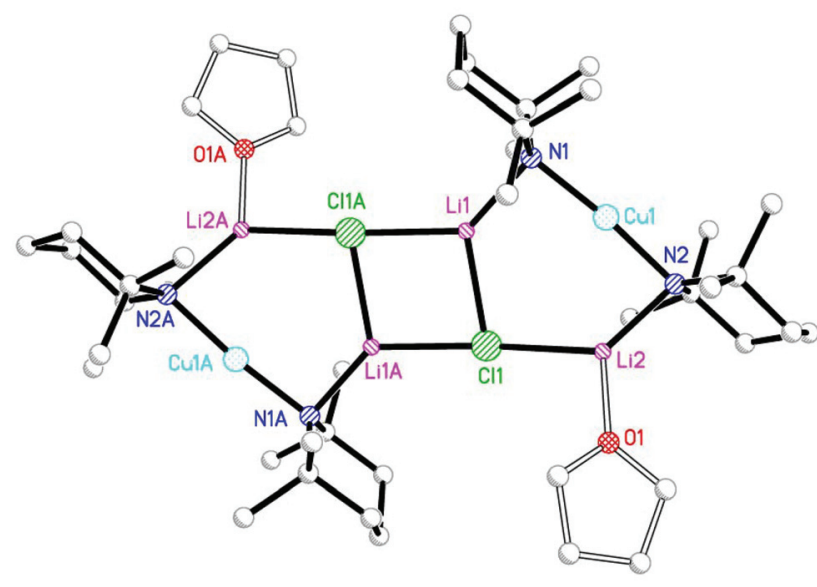

Fig. 4 Structure ( $\mathrm{H}$ atoms omitted) of the crystallographic dimer of chloride 9.

formed (TMP) $)_{2} \mathrm{CuLi}$ (section 3) produced distinctly inferior reactivity. Overall, therefore, it could be posited that the optimal reagent adhered to the putative formulation (TMP) $)_{2} \mathrm{Cu}(\mathrm{Cl}) \mathrm{Li}_{2}{ }^{28}$ Corroboration came from subsequent studies conducted on crystalline material isolated from reaction of TMPLi with $\mathrm{CuCl}$ in THF. The resulting structure, of $(\mathrm{TMP})_{2} \mathrm{Cu}(\mathrm{Cl}) \mathrm{Li}_{2} \cdot \mathrm{THF} 9$ (Fig. 4), revealed a solid-state dimer based on a (ClLi $)_{2}$ core. $^{29}$ Superficially, the structure is highly analogous to that revealed by both 7 (albeit the Cu-containing rings are now 6- rather than 7 -membered) and $\mathbf{8}$ (albeit the structure is dimeric). As with these structures, 9 incorporates lower-order, near-linear $\mathrm{Cu}$ centres.

Comparative research on the synthesis of diaryl ketones using lithiocuprates prepared with $\mathrm{LiCl}$ and $\mathrm{LiBr}$ has been reported and is discussed in section 3.3. As with chloridebased 9, the dimer of $(\mathrm{TMP})_{2} \mathrm{Cu}(\mathrm{Br}) \mathrm{Li}_{2} \cdot \mathrm{THF} 10$ proved straightforward to isolate and characterise. ${ }^{30}$ The dimeric structure of this species contrasted with that noted previously for monomeric $\left(\mathrm{C}_{6} \mathrm{H}_{4}\left\{\mathrm{CH}_{2} \mathrm{NMeCH}_{2} \mathrm{CH}_{2} \mathrm{NMe}_{2}\right\}-2\right)_{2} \mathrm{Cu}(\mathrm{Br}) \mathrm{Li}_{2}$ 11 in which aggregation was inhibited by internal coordination of the alkali metals (Fig. 5). ${ }^{31}$ Finally, the iodide analogue of 9 and 10, (TMP) $)_{2} \mathrm{Cu}(\mathrm{I}) \mathrm{Li}_{2} \cdot \mathrm{THF} 12$, was also prepared (Fig. 6). ${ }^{32}$

The Cu-containing metallocycles in 7 are 7 -membered and show near perpendicular geometry at the bridging nitrogen centres, there being only a slight asymmetry here (N1/2-Li2/1

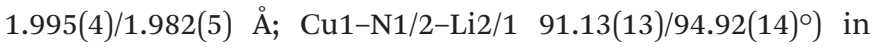
spite of the fact that the two alkali metal centres in the ring have significantly different coordination environments. On moving to the chloride 9 and bromide 10, the most immediate structural change is that the cuprate moieties yield 6-membered rings which are highly asymmetric. This is revealed by the remarkably different $\mathrm{N}$-Li distances and bridging angles at N. For example, in $\mathbf{1 0}$ the unsolvated alkali metal ion participates in a relatively short interaction with TMP (N2-Li2 1.963(4) Å compares with N1-Li1(THF) 2.002(5) ̊). In a similar vein, the bridging angle at $\mathrm{N} 2$ is expanded relative to

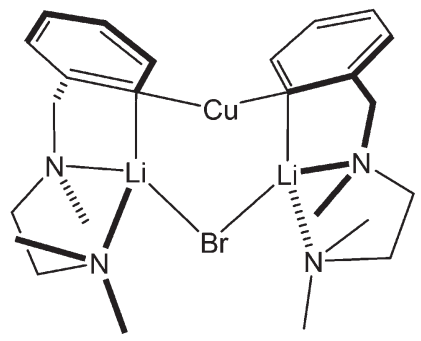

Fig. 5 Monomeric structure of internally stabilized cuprate 11.

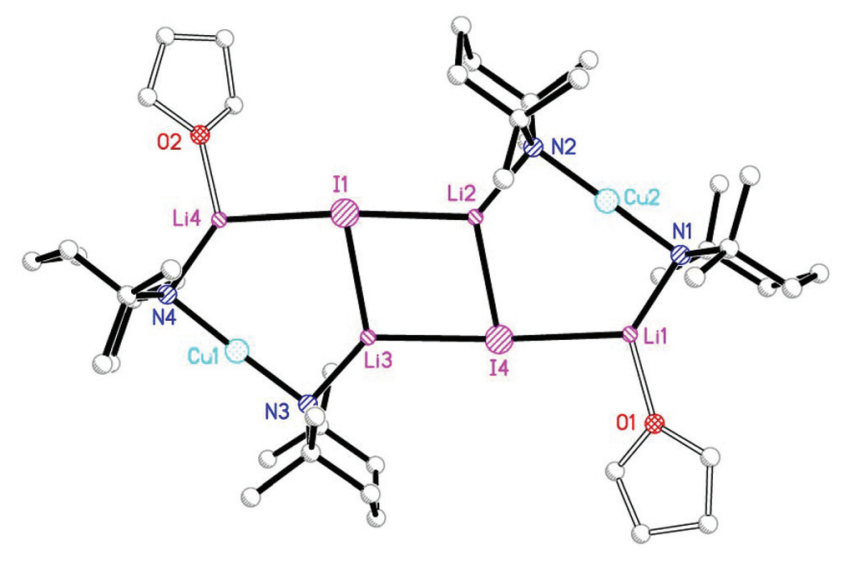

Fig. 6 Structure of the non-centrosymmetric dimer of $\mathbf{1 2}$ ( $\mathrm{H}$ atoms and iodide centre positional disorder omitted).

that at $\mathrm{N} 1\left(c f .92 .81(14)\right.$ and $85.45(13)^{\circ}$, respectively). The same pattern is clearly seen for chloride 9, but moving to iodide 12, patterns in the bonding parameters depart somewhat from those seen in $\mathbf{9}$ and $\mathbf{1 0}$ in that the extreme asymmetry seen for the 6-membered cuprate metallocycles in the chloride and bromide dimers is present to a much reduced extent. Thus, although $\mathrm{N}-\mathrm{Li}$ is significantly shorter than $\mathrm{N}-\mathrm{Li}$-THF, four very similar $\mathrm{Cu}-\mathrm{N}-\mathrm{Li}$ bond angles (range 93.11 (18)-94.63(18) ${ }^{\circ}$ are seen. The N-Li interactions reveal a final point about the cuprate metallocycles in 12. At $1.965 \AA$ (mean) these are shorter than those in 7 (mean $1.989 \AA$ ), consistent with the preference of hard $\mathrm{Li}^{+}$for hard nitrogen over soft iodide.

In a similar vein to the synthesis of THF-solvates 7, 9, 10 and 12, the extension in Li-X bonding interactions as Group 17 is descended is noted also for the employment of $\mathrm{OEt}_{2}$ as Lewis base. The resulting lithiocuprates 13-16 (for $\mathrm{X}=\mathrm{CN}, \mathrm{Cl}$, $\mathrm{Br}$ and I, respectively; Fig. 7) have all been found to be essentially analogous to their THF-solvated counterparts. Poor quality data prevents detailed discussion of $\mathbf{1 3}$. However, analysis of the bonding in 14-16 reveals that, in comparison to the THF-solvates discussed above, their 6-membered rings show a higher level of symmetry, in spite of the clearly different alkali metal environments therein. 


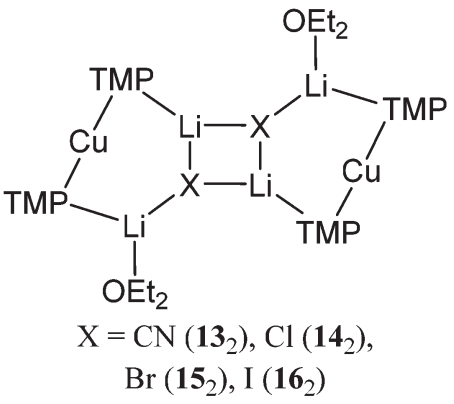

Fig. 7 The solid-state formulation of diethyl ether-solvated 13-16.

\subsection{Gilman reagents}

Of course, as noted above, the first lithium cuprates to be prepared were not Lipshutz or Lipshutz-type but rather were considered to have the formulation $\mathrm{R}_{2} \mathrm{CuLi}($ e.g. $\mathrm{R}=\mathrm{Me} \mathbf{1})$. These have come to be known as Gilman reagents. In the context of deprotonative cupration, we describe elsewhere in this article (section 3.2) synthetic transformations done in hydrocarbon media that drive the removal of lithium salt from the reaction and which, therefore, promote Gilman cuprate activity. Specifically, lithium cuprates of the type $\mathrm{R}_{m} \mathrm{Cu}\left(\mathrm{NR}_{2}\right)_{n} \mathrm{Li}(m=1, n=1$; $m=0, n=2)$ are relevant to the organic transformations described here and these have not been thoroughly studied historically. The structural possibilities for these systems have only begun to be elucidated in the last few years and this has led the field to be the subject of review. ${ }^{33}$

Concerning lithium (TMP)cuprates, the treatment of TMPLi with CuCN and various $\mathrm{CuX}(\mathrm{X}=$ halide) reagents in THF has been described above as yielding 7 and 9, 10 and 12 (and their $\mathrm{OEt}_{2}$-solvated analogues 13-16). Bis(amido)cuprate formulations such as these have been shown (see section 3.2) to effect DoC with the TMP ligand acting as a base, enabling homocoupling to give 2,2-biaryls. However, the solution structures of these systems are still not fully established. This study involves not only the elucidation of aggregation state but also understanding of the affinity of (TMP) ${ }_{2} \mathrm{CuLi}$ for LiX; that is, the question of whether a Lipshutz-type cuprate may interconvert with a Gilman cuprate through the inclusion/exclusion of LiX. As the discussion of recent theoretical studies in section 4 makes clear, this question is one of enormous importance in understanding DoC reactivity. By way of an example, whereas the previous sections of this structural discussion focus on Lipshutz cuprates $(\mathrm{TMP})_{2} \mathrm{Cu}(\mathrm{CN}) \mathrm{Li}_{2} \cdot \mathrm{S} 7$ and $13\left(\mathrm{~S}=\mathrm{THF}, \mathrm{OEt}_{2}\right)$ and also Lipshutz-type reagents (TMP) $)_{2} \mathrm{Cu}(\mathrm{X}) \mathrm{Li}_{2} \cdot \mathrm{S} \mathbf{9 , 1 0 , 1 2}$ and 14-16 ( $\mathrm{X}$ = halide; $\mathrm{S}=\mathrm{THF}, \mathrm{OEt}_{2}$ ), a bis(TMP) Gilman cuprate has been prepared using remarkably similar chemistry. Hence, the reaction of TMPLi with CuI in the presence of THF (in the ratio $2: 1: 1$ ) has given (TMP) ${ }_{2} \mathrm{Cu}(\mathrm{I}) \mathrm{Li}_{2} \cdot \mathrm{THF} 12$ (see above). In contrast, similar reaction in the absence of THF represents a strategy designed to allow the precipitation and removal of lithium halide. This has borne fruit as discussed in the organic synthesis section of this review, where the efficiency with which deprotonative transformations have been achieved was found to correlate with whether or not lithium salt was

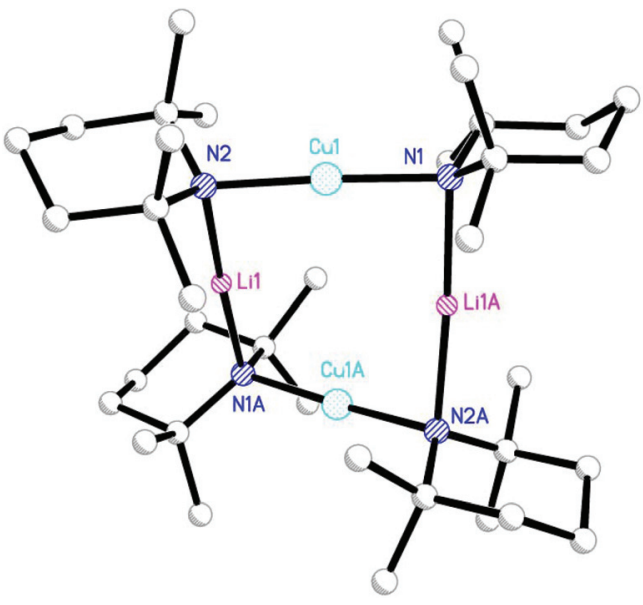

Fig. 8 The dimer of 17 with $\mathrm{H}$ atoms omitted.

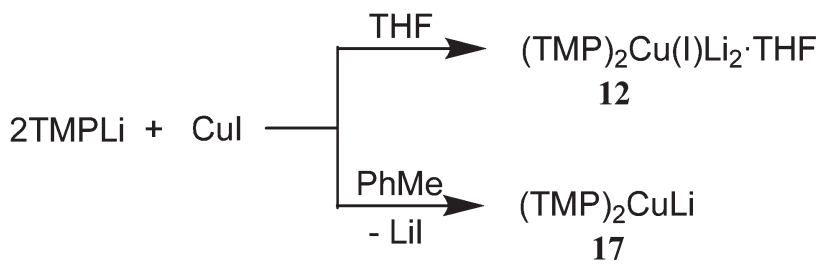

Scheme 3 Formation of Gilman reagent 17 accompanied by the expulsion of lithium iodide, which precipitates in the absence of donor solvent and so avoids the formation of 12 .

abstracted from the system. In the present case, LiI precipitation facilitated the isolation of Gilman bis(amido)cuprate $(\mathrm{TMP})_{2} \mathrm{CuLi} 17 .^{32}$ Crystallography reveals a cyclic dimer in the solid-state (Fig. 8 and Scheme 3). In spite of the known ability of organolithium and organocopper species to exchange (see below) the metal sites in the dimer of $\mathbf{1 7}$ revealed hardly any substitutional disorder and the structure compares closely with the 8-membered metallocyclic dimer of $\mathrm{MesNHCu}(\mathrm{PhNH})$ Li·DME 18 (DME = 1,2-dimethoxyethane; Fig. 9). ${ }^{27}$ The isolation of 17 proved useful because it provided pure Gilman bis(TMP)cuprate for comparative tests of Lipshutz-type and Gilman cuprates in organic synthesis (section 3.3).

As noted above, the article in which DoC was first presented discussed the synthesis of homocoupled products. ${ }^{6}$ However,

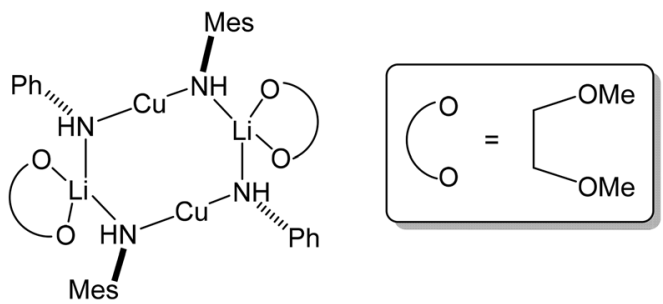

Fig. 9 The 8-membered metallocyclic dimer of $\mathrm{MesNHCu}(\mathrm{PhNH})$ Li.DME 18. 


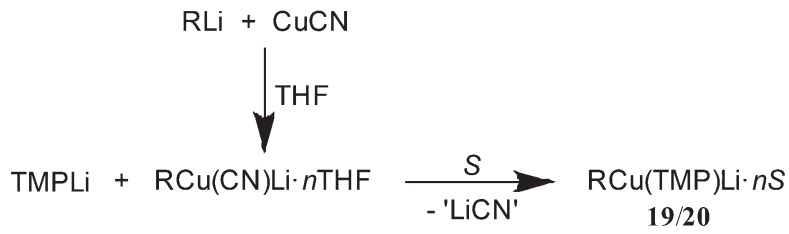

Scheme 4 The formation of Gilman cuprates $19(\mathrm{R}=\mathrm{Ph}, n=3, \mathrm{~S}=$ THF) and 20 (R = Me, $n=1, \mathrm{~S}=\mathrm{TMEDA})$.

it was also suggested that the synthesis of heterocoupled products could be achieved by using the putative cuprates R(TMP)$\mathrm{Cu}(\mathrm{CN}) \mathrm{Li}_{2}(\mathrm{R}=$ alkyl, aryl $)$. To investigate, $\mathrm{CuCN}$ was treated with RLi and TMPLi. For R $=\mathrm{Ph}$ crystallography now revealed the formation of monomeric PhCu(TMP)Li·3THF 19 (Scheme 4 and Fig. 10). In a similar vein, the ability of putative cuprate $\mathrm{Me}(\mathrm{TMP}) \mathrm{Cu}(\mathrm{CN}) \mathrm{Li}_{2}$ to achieve products in which aryl substrates were methylated led to investigation of the MeLiCuCN-TMPLi mixture. With crystallisation being aided by the introduction of TMEDA $\left(N, N, N^{\prime}, N^{\prime}\right.$-tetramethylethylenediamine) it proved possible to isolate cuprate $\mathrm{MeCu}(\mathrm{TMP})$ Li-TMEDA $20 .{ }^{34}$

The observation of a terminal $\mathrm{Cu}-\mathrm{Ph}$ interaction in 19 is unusual. Whilst it is known from other types of organocopper compound ${ }^{35}$ the only lithiocuprate examples are $\left[\mathrm{Ph}_{2} \mathrm{Cu}\right]^{-}[\mathrm{Li} \cdot 2(12-\mathrm{crown}-4)]^{+} \quad 21$ and $\left\{\left[\mathrm{Ph}_{2} \mathrm{Cu}\right]^{-}\right\}_{2}\left[\left(\mathrm{Cu}\left(\cdot 2 \mathrm{PPh}_{3}\right)-\right.\right.$ $\left.\left.\{(\mathrm{CN}) \mathrm{Li}\}_{2} \cdot 5 \mathrm{THF}\right)_{2}\right]^{2+} \mathbf{2 2},{ }^{36}$ whilst the remaining reported terminal $\mathrm{Cu}-\mathrm{Ar}$ bonds in this area all feature bulky Mes, ${ }^{37} \mathrm{C}_{6} \mathrm{H}_{3}-2,6-$ $\mathrm{Mes}_{2},{ }^{38} \mathrm{C}_{6} \mathrm{H}_{3}-2,6-\mathrm{Trip}_{2}$ (Trip $\left.=\mathrm{C}_{6} \mathrm{H}_{2}-2,4,6-\mathrm{Pr}_{3}{ }_{3}\right)^{39}$ or ferrocenyl groups. ${ }^{40}$ Moving to the similarly underpopulated field of heteroleptic organyl(amido)cuprate structural chemistry ${ }^{41} 19$ fails to demonstrate the aggregation behaviour of aryl(amido)cuprate $\mathrm{MesCu}\left(\mathrm{NBz}_{2}\right) \mathrm{Li} 23$ (Bz = benzyl; Fig. 11$),{ }^{42}$ the head-totail dimeric structure of which confirmed theoretical expectations. Even in solution, the dimer showed negligible signs of deaggregation. ${ }^{43}$ However, it was clear that $\mathbf{2 3}$ represented the only compound isolable from a Schlenk equilibrium involving a variety of structural isomers in solution. ${ }^{42}$ Methylcuprate ions are even less well documented, having been noted only in

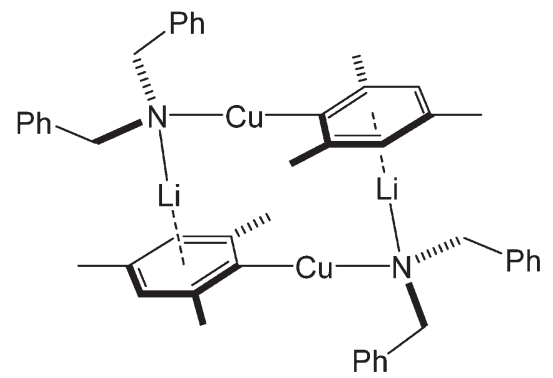

Fig. 11 The dimeric aggregate of $\mathrm{MesCu}\left(\mathrm{NBz}_{2}\right) \mathrm{Li} 23$.

$\left[\mathrm{Me}_{2} \mathrm{Cu}\right]^{-}[\mathrm{Li} \cdot n \mathrm{~S}]^{+}(24 n=2, \mathrm{~S}=12$-crown- $4 ; 25 n=3, \mathrm{~S}=$ $\mathrm{DME})^{36,44}$ and the alkyl(phosphido)cuprate $\operatorname{MeCu}\left\{\mathrm{P}\left(\mathrm{Bu}^{t}\right)_{2}\right\}$ Li.3THF 26 (Fig. 12). ${ }^{45}$ The observation of a single $\mathrm{N}$-atom bridge between metals in $\mathbf{1 9}$ and 20 is interesting in the wider context of synergic amido-reagents. Hitherto, these have frequently revealed metallocyclic $\mathrm{M}(\mu-\mathrm{R})(\mu-\mathrm{N}) \mathrm{Li}$ cores, even after isolation from (and including within their structures) Lewis base media. Hence, metallocycle formation has proved unambiguous in aluminates (where $\mathrm{M}$ is Group 13) ${ }^{46}$ and zincates (where $\mathbf{M}$ is Group 12 and therefore stands comparison with Group 2). ${ }^{47}$ However, in recently observed manganates (where $\mathrm{M}$ is Group 7 and therefore stands comparison with Groups 2 and 12), ${ }^{48}$ closure of the ring via the formation of an agostic bridge $^{49}$ to match the amido one was much more ambiguous (Scheme 5). Finally, in the present case (where M is Group 11

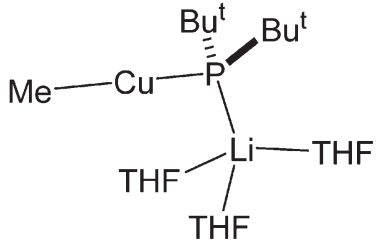

Fig. 12 Alkyl(phosphido)cuprate 26.
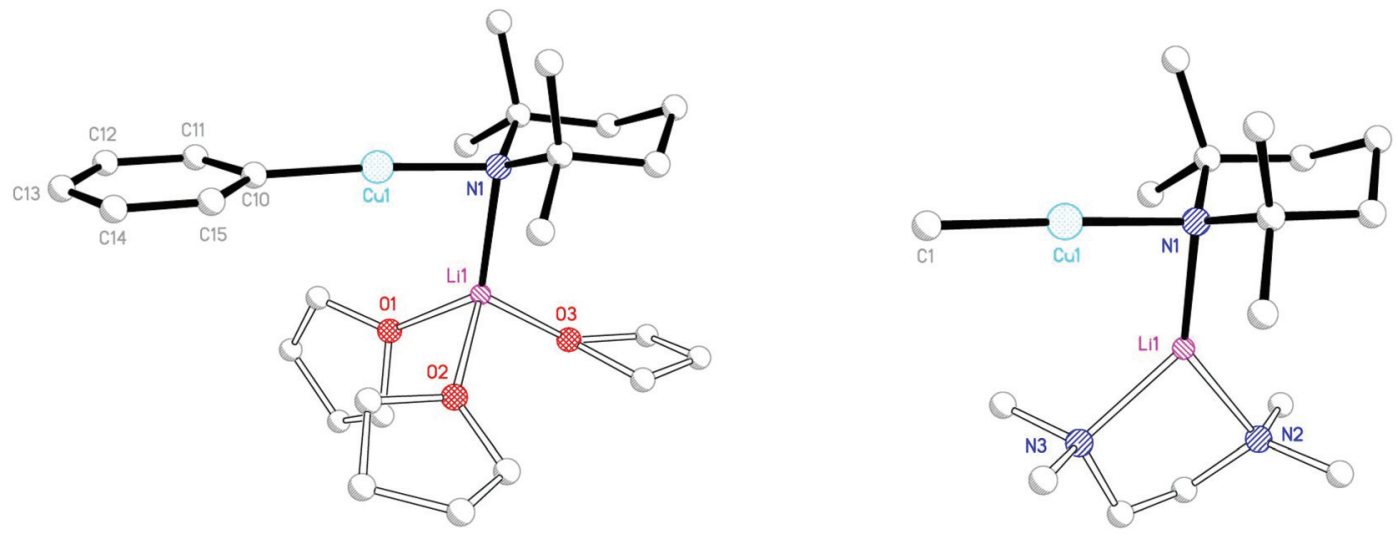

Fig. 10 Structures of 19 (left) and 20 (right) with $\mathrm{H}$ atoms and disorder omitted. 


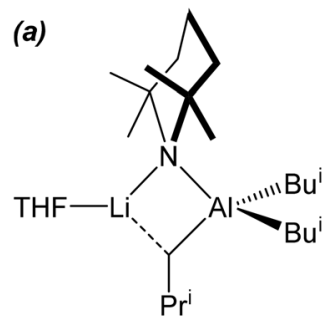

(c)

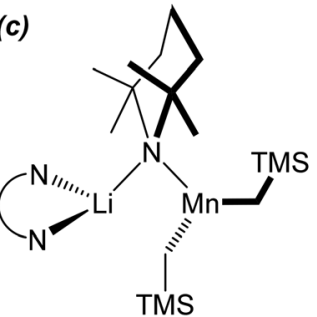

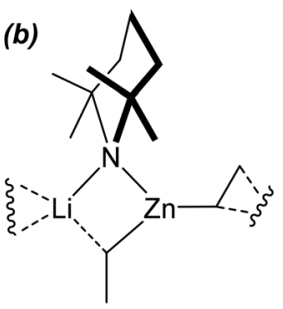

(d)

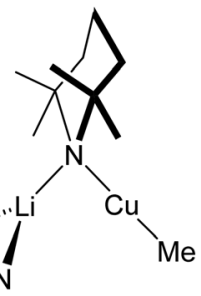

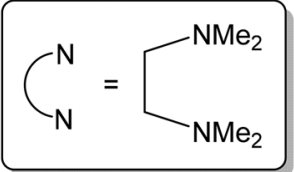

Scheme 5 The cores of synergic bases containing $\mathrm{Al}, \mathrm{Zn}, \mathrm{Mn}$ and $\mathrm{Cu}$. (a) and (b) metallocycle completion via short Li $\ldots \mathrm{C}$ approaches of $2.258(4)^{46 b}$ and $2.374(3)^{47 d} \AA$, respectively; (c) the central ring fails to form, with Li...C relatively extended at $2.742(6) \AA_{;}^{47 a}$ (d) Cu exhibits a near linear geometry, precluding agostic stabilisation of the alkali metal.

and stands comparison with Group 1) the essentially linear geometry at $\mathrm{Cu}^{50}$ clearly prevents the formation of a metallocycle.

\subsection{Amidocuprate adducts: the abstraction of lithium halide}

Recent studies in the rapidly evolving field of so-called synergic base chemistry have focused on minimizing the costs of reagent preparations. ${ }^{51}$ This has led to the deployment of DMPH (= cis-2,6-dimethylpiperidine) in place of TMPH. At time of writing, the relative costs of these two amines are $£ 2.62 \mathrm{~g}^{-1}$ (TMPH) and $£ 0.20 \mathrm{~g}^{-1}$ (DMPH), making the potential savings of developing DMP-ligated synergic bases significant. ${ }^{52}$ The adoption of much cheaper amide ligands has gone handin-hand with the removal of some of the significant steric hindrance associated with TMP ligands, allowing fundamental changes to the orientation of the amide group and enabling the observation of new amidocuprate structure-types. ${ }^{53}$

As noted above, the use of diethyl ether as a solvent enabled the synthesis and isolation of Lipshutz-type (TMP) ${ }_{2} \mathrm{Cu}$ $(\mathrm{Cl}) \mathrm{Li}_{2} \cdot \mathrm{OEt}_{2}$ 14. However, upon exchanging the amide component for DMP a dramatic effect was observed. Initially, the combination of DMPLi with $\mathrm{CuCl}$ was attempted in limited (2 equiv.) $\mathrm{Et}_{2} \mathrm{O}$. Reaction led to the isolation of crystalline material that analyzed as a triangulated structure based on a $\mathrm{LiCl}$ core and having the formulation [(DMP $\left.)_{4} \mathrm{CuM}\left(\mathrm{Li} \cdot \mathrm{OEt}_{2}\right)_{2}\right]$ LiCl 27 where optimal refinement of multiple crystallographic datasets suggested a pattern of disorder consistent with $\mathbf{M}=$ $0.3: 0.7 \mathrm{Cu}: \mathrm{Li}$ (Scheme 6).

The disorder at one of the metal sites complicates interpretation of the nature of 27 but is interesting because it is suggestive of the phenomenon of exchange between organocopper and organolithium species that has been directly observed spectroscopically in the past. ${ }^{54}$ Overall, two views of 27 present themselves. For $\mathrm{M}=\mathrm{Li}$ the complex could be viewed as a $1: 2$

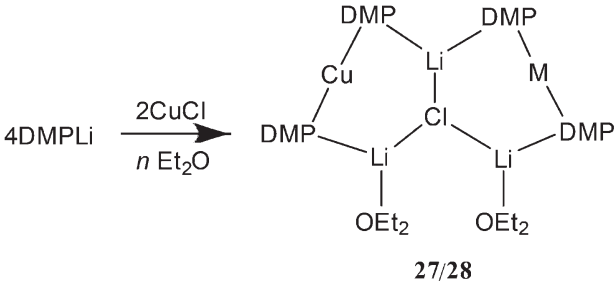

Scheme 6 Synthesis of $27(\mathrm{M}=30: 70 \mathrm{Cu}: \mathrm{Li}, n=2$ equiv. wrt $\mathrm{Cu})$ and $28(\mathrm{M}=\mathrm{Cu}, n=$ bulk).

adduct between Lipshutz-type (DMP $)_{2} \mathrm{Cu}(\mathrm{Cl}) \mathrm{Li}_{2}$ and DMPLi. However, for $\mathrm{M}=\mathrm{Cu}$ it can be argued that 27 represents an adduct between Gilman cuprate (DMP) $)_{2} \mathrm{CuLi} \cdot \mathrm{OEt}_{2}$ and Lipshutz-type cuprate $(\mathrm{DMP})_{2} \mathrm{Cu}(\mathrm{Cl}) \mathrm{Li}_{2} \cdot \mathrm{OEt}_{2}$. To overcome this ambiguity and to remove the observed metal disorder, solvent mixtures were varied. Ultimately, the use of bulk $\mathrm{Et}_{2} \mathrm{O}$ served to reinforce the interpretation of 27 as an adduct between two cuprates, with $\left[(\mathrm{DMP})_{2} \mathrm{CuLi} \cdot \mathrm{OEt}_{2}\right]_{2} \mathrm{LiCl} \mathbf{2 8}$ being isolated (Scheme 6 and Fig. 13).

It proved possible to replicate the formation of 28 using $\mathrm{CuBr}$ (Scheme 7 and Fig. 14). A number of iterations were required to allow the isolation of $\left[(\mathrm{DMP})_{2} \mathrm{CuLi} \cdot \mathrm{OEt}_{2}\right]_{2} \mathrm{LiBr} 29$. Hence, the reaction of $\mathrm{Bu}^{n} \mathrm{Li}$ (1 eq. wrt amine) with $\mathrm{DMPH}$ to give DMPLi was initially affected in limited Lewis base (2 equiv. $\left.\mathrm{Et}_{2} \mathrm{O}\right)$. However, this afforded $\left[(\mathrm{DMP})_{2} \mathrm{Cu}\right.$ $\left.\mathrm{Li} \cdot \mathrm{OEt}_{2}\right]_{1.45}\left[(\mathrm{DMP})_{2} \mathrm{CuLi} \cdot \mathrm{DMPH}\right]_{0.55} \mathrm{LiBr} 30$. This problem could be overcome, in two ways: the use of excess $\mathrm{Bu}^{n} \mathrm{Li}$ (wrt amine) or the deployment of bulk $\mathrm{Et}_{2} \mathrm{O}$ as solvent. Variation in solvent conditions has therefore been shown to have an effect on the production of adducts 28 and 29, with bulk $\mathrm{Et}_{2} \mathrm{O}$ working best for $\mathrm{X}=\mathrm{Cl}$ and either bulk or 2 equiv. $\mathrm{Et}_{2} \mathrm{O}$ best for $\mathrm{X}=\mathrm{Br}$. Attempts to extend the new chemistry to $\mathrm{X}=\mathrm{I}$ demonstrated a need to more stringently control the quantity of $\mathrm{Et}_{2} \mathrm{O}$, with the 


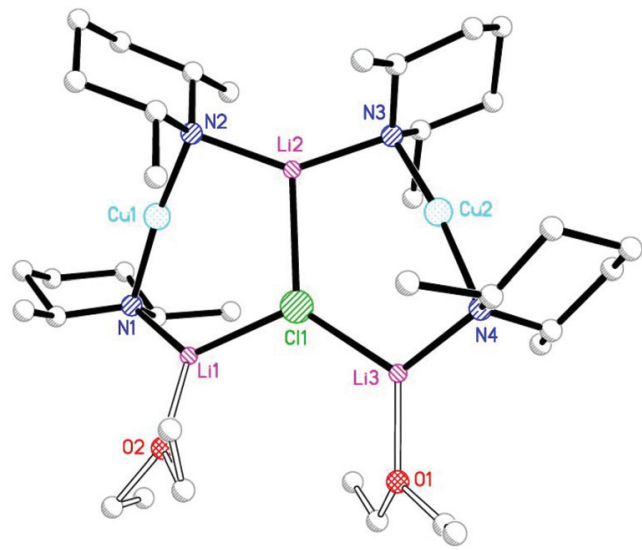

Fig. 13 Adduct $\left[(\mathrm{DMP})_{2} \mathrm{CuLi} \cdot \mathrm{OEt}_{2}\right]_{2} \mathrm{LiCl}$ 28. $\mathrm{H}$ atoms and minor $\mathrm{Et}_{2} \mathrm{O}$ disorder omitted.

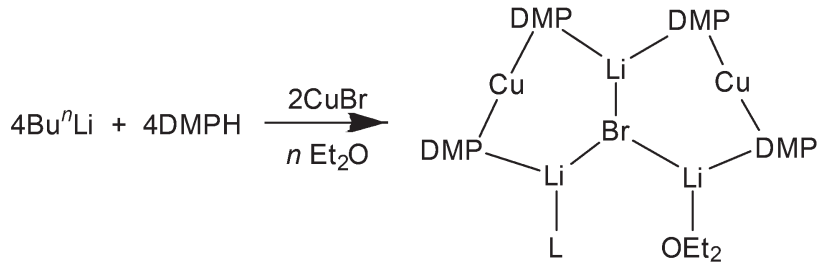

29/30

Scheme 7 Synthesis of $29\left(n=\right.$ bulk; $\left.\mathrm{L}=\mathrm{OEt}_{2}\right)$ and $30(n=2$ equiv. wrt $\left.\mathrm{Cu} ; \mathrm{L}=\left(\mathrm{OEt} \mathrm{t}_{2}\right)_{0.45}(\mathrm{DMPH})_{0.55}\right)$.

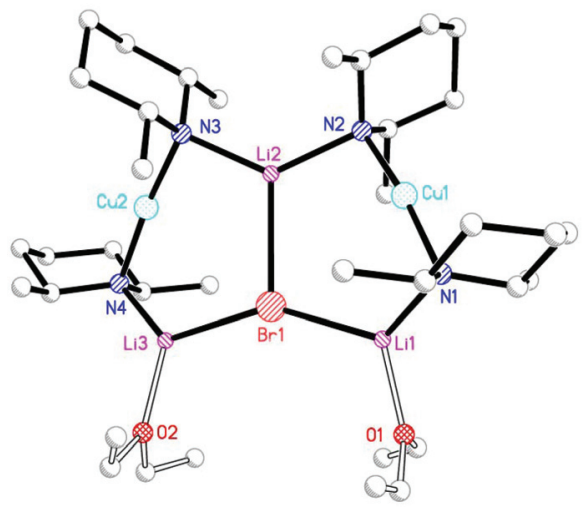

Fig. 14 Adduct [(DMP $\left.{ }_{2} \mathrm{CuLi} \mathrm{OEt}_{2}\right]_{2} \mathrm{LiBr} 29 . \mathrm{H}$ atoms omitted.

use of 1 equiv. wrt $\mathrm{Cu}$ yielding $\left[(\mathrm{DMP})_{2} \mathrm{CuLi} \cdot \mathrm{OEt}_{2}\right]_{2} \mathrm{LiI} \quad 31$ (Scheme 8 and Fig. 15).

The metal disorder in $\mathbf{2 7}$ notwithstanding, the adduct structures all approximate to $C_{2}$ symmetry about a core lithium halide axis. The metal-halide bonds in adducts 28,29 and 31 extend as Group 17 is descended. Though this is unsurprising in itself, the extension proves not to be consistent. At 1.10, the ratio between $\mathrm{Li} 2-\mathrm{X}$ and the mean of $\mathrm{Li} 1 / 3-\mathrm{X}$ is greatest for $\mathrm{X}=$ I. For both the smaller halides it is 1.04 , and this can be rationalised in terms of the relative preference of the alkali metal for interaction with hard and soft donors. The extension

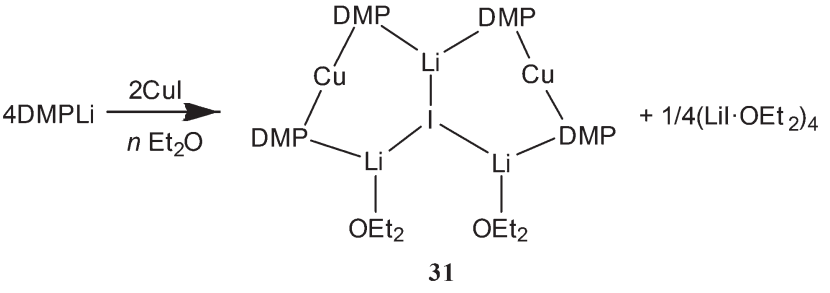

Scheme 8 Synthesis of 31 ( $n=1$ equiv. wrt $\mathrm{Cu}$ ).

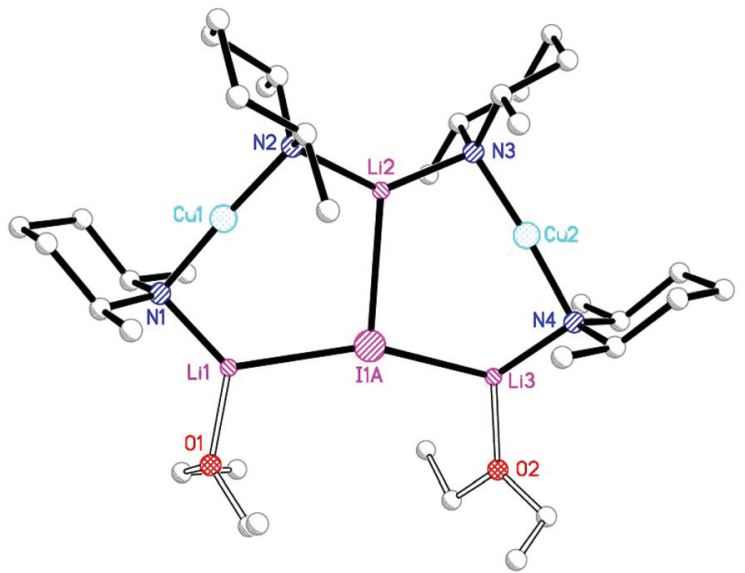

Fig. 15 Adduct $\left[(\mathrm{DMP})_{2} \mathrm{CuLi}^{\mathrm{O}} \mathrm{OEt}{ }_{2}\right]_{2} \mathrm{Lil} 31 . \mathrm{H}$ atoms and minor disorder omitted.

of metal-nitrogen bonding as a function of halide hardness is also reflected in the $\mathrm{Li} 2-\mathrm{N}-\mathrm{Cu}$ angles, which increase in response to the presence of a softer halide. Moreover, a persistent feature of these ether-solvated adducts is distinct asymmetry in both the $\mathrm{N}-\mathrm{Li}$ bond lengths and bridging angles at nitrogen. This contrasts with the much more symmetrical rings in diethyl ether solvates 14-16 (see section 2.2) in spite of the observation of essentially the same Cu-containing 6-membered rings in which only one of the two $\mathrm{Li}^{+}$ions is solvated by $\mathrm{OEt}_{2}$. For example, angles at either $\mathrm{N}$ in a 6-membered ring deviate by $<3^{\circ}$ in 14-16 whereas in adducts $\mathbf{2 8}, \mathbf{2 9}$ and 31 they differ by $4.2-6.6^{\circ}$.

The observation of a transition in structure-type from Lipshutz-type dimers to adducts is intriguing. It is also potentially important in terms of rationalising DoC. As is explained in sections 3 and 4 of this review, DoC requires reagents that are formally Lipshutz(-type) but which may generate active monomeric Gilman bases. This revelation - that generation of the active base takes place in situ - has prompted an examination of structure-types synonymous with the abstraction of LiX from Lipshutz-type dimers, and it is clear that adducts of the type now discovered can be viewed in these terms.

Since 14-16 and 28, 29 and 31 are all (mono)ether solvates it stands to reason that the choice of amide ligand must be having a significant effect on structure. This idea bears fruit if the steric constraints associated with TMP and DMP ligands are considered. In, for example, the ether-solvated Lipshutztype chloride dimer of (TMP) ${ }_{2} \mathrm{Cu}(\mathrm{Cl}) \mathrm{Li}_{2} \cdot \mathrm{OEt}_{2} \mathbf{1 4}$ it is clear that 

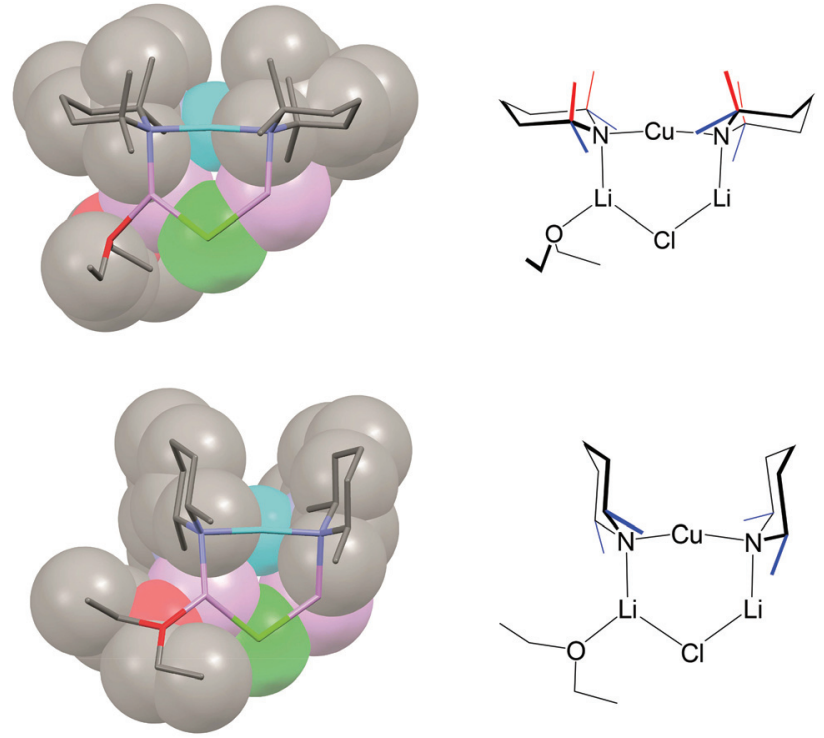

Fig. 16 Structures of the 6-membered heterocyclic Lipshutz-type components that form parts of the dimer of TMP-based chloride 14 (top) and DMP-based adduct 28 (bottom). In 14 the piperidide ligands lie flat in order that the axial Me-groups (red) do not sterically interact. In 28 only equatorial Me-groups are present (blue) and the piperidide ligands can reside face-on.

the two TMP ligands bonded to either $\mathrm{Cu}$ atom project away from one another so that the two 6-membered piperidide rings lie in a fashion best described as endo,endo relative the $(\mathrm{ClLi})_{2}$ aggregate core (Fig. 16, top). The need for this to happen is rationalised by considering the positions of the 8 methyl groups associated with this pair of ligands. Four of these methyl groups (blue in Fig. 16) exhibit equatorial positions relative to the piperidide rings while the remaining four are axially disposed. Clearly, the axial Me-groups (red in Fig. 16) would interact with each other in the event that the piperidide ligands, instead of lying endo,endo, adopted an exo,exo (or face-on) arrangement. Such an arrangement is, however, enabled by the replacement of TMP by DMP in $\mathbf{2 8}$. In this case the two DMP ligands bonded to a given $\mathrm{Cu}$ centre can now exhibit a face-on motif that is exo,exo with respect to the structure core (the $\mathrm{LiCl}$ vector in 28) without there being steric interaction between the methyl groups (Fig. 16, bottom).

The fact that, in the absence of steric interaction between methyl groups, the piperidide rings adopt a face-on configuration vexes the question: is this a preferred arrangement? This unfortunately cannot be answered by an analysis of the adduct structures reported here because the ability of the piperidide ligands to adopt a face-on orientation confers significant stability on the structure in terms of steric interactions not only within the monomeric building block but also between Lipshutz-type and Gilman components of the triangulated structure. As suggested by Fig. 17, the exo,exo orientation of the amide ligands allows steric congestion at the unsolvated $\mathrm{Li}^{+}$ (Li2 in Fig. 13-15) to be acceptable. An endo,endo structure would be precluded for obvious reasons while the inability to observe exo,exo TMP-based analogues of DMP-based adducts 28-31 may also be explicable on steric grounds.

The adducts so far prepared have demonstrated a significant tendency to retain their structures in hydrocarbon solution. This has best been evidenced by ${ }^{7} \mathrm{Li}$ NMR spectroscopy. For the chloride adduct $\left[(\mathrm{DMP})_{2} \mathrm{CuLi} \cdot \mathrm{OEt}_{2}\right]_{2} \mathrm{LiCl} 28$ the bis(amide)-complexed alkali metal is seen at more positive chemical shift ( $\delta 1.83 \mathrm{ppm})$ than the externally solvated metal ions, which resonate at $\delta 1.48 \mathrm{ppm}$, with the two peaks exhibiting a $1: 2$ ratio. Identical trends are observed for 29 and 31. The incompletely deprotonated complex $\left[(\mathrm{DMP})_{2} \mathrm{Cu}-\right.$ $\left.\mathrm{Li} \cdot \mathrm{OEt}_{2}\right]_{1.45}\left[(\mathrm{DMP})_{2} \mathrm{CuLi} \cdot \mathrm{DMPH}\right]_{0.55} \mathrm{LiBr} 30$ presents a yet more interesting spectrum. The unique bis(amide)-complexed alkali metal ion is seen at $\delta 1.83 \mathrm{ppm}$, while equivalent integration signals are now also seen at $\delta 1.66 \mathrm{ppm}$ and $\delta 1.48 \mathrm{ppm}$, and these are attributable to $\mathrm{Li} \cdot \mathrm{DMPH}$ and $\mathrm{Li} \cdot \mathrm{OEt}_{2}$.

Lastly, recent work on the new class of cuprate adduct discussed here has sought to extend our understanding of the generality with which they form by varying the solvent. Reaction of DMPLi with CuBr followed by recrystallization in the presence of THF has afforded $\left[(\mathrm{DMP})_{2} \mathrm{CuLi} \cdot 2 \mathrm{THF}\right]_{2} \mathrm{LiBr} 32$ (Fig. 18 and Scheme 9). In so doing the ability of THF to furnish an adduct has been demonstrated along with the observation that much more extensive solvation of the alkali metals is possible in these structures.
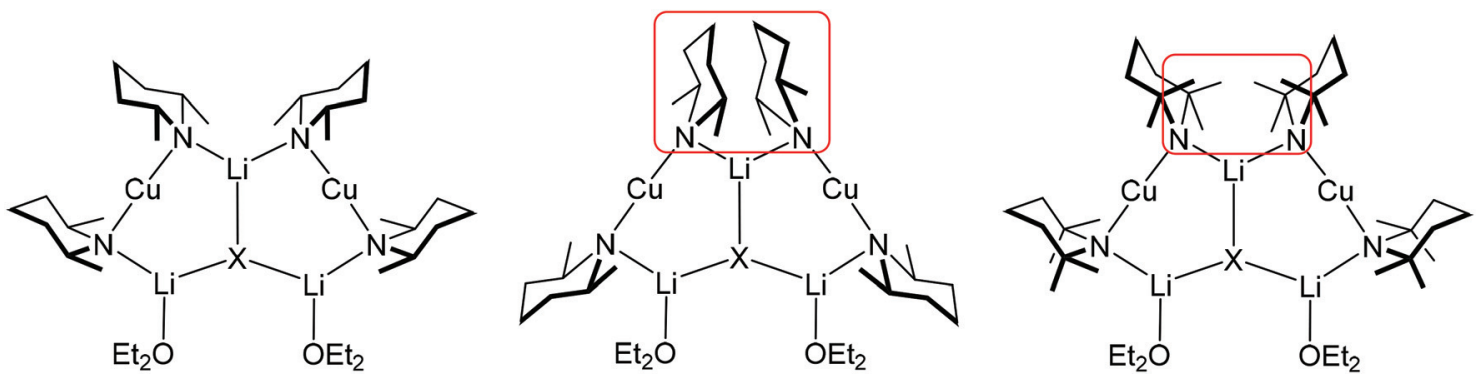

Fig. 17 A schematic of the observed exo,exo (left) and less favoured endo,endo forms (middle) of DMP-based adducts akin to 28-31 clarifies why only the first option has been seen experimentally. Steric interaction at the structure apex may also explain why TMP-based adducts have not so far been seen (right). 


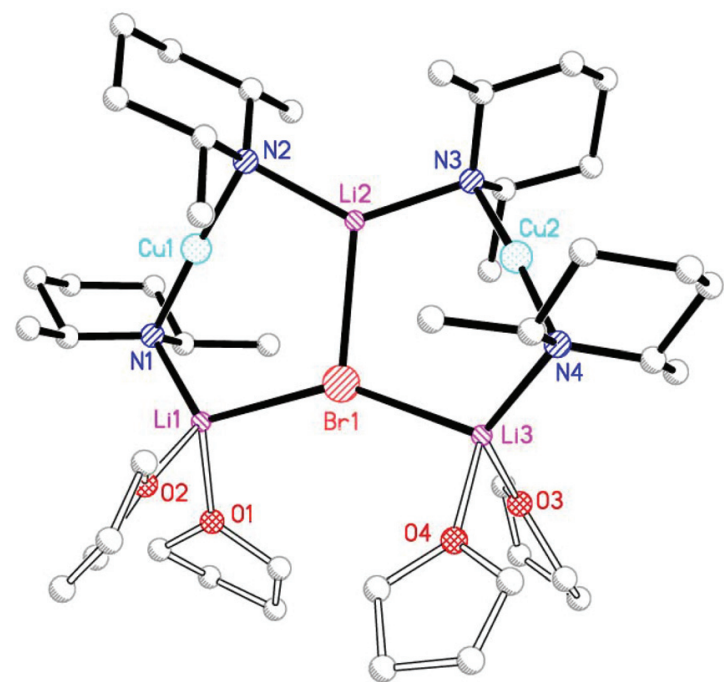

Fig. 18 Adduct $\left[(\mathrm{DMP})_{2} \mathrm{CuLi} \cdot 2 \mathrm{THF}\right]_{2} \mathrm{LiBr} 32 \mathrm{H}$ atoms omitted.

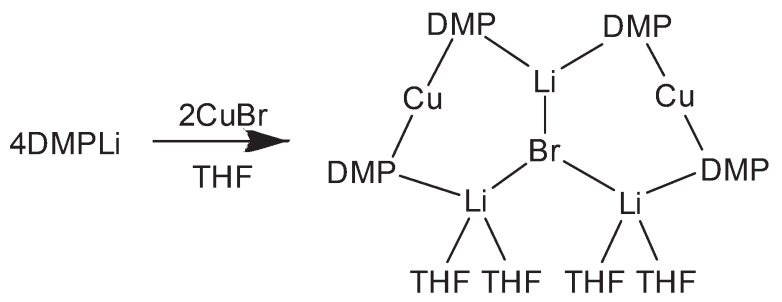

32

Scheme 9 Synthesis of adduct 32.

\section{Recent applications of lithium cuprates in organic synthesis}

\subsection{Gilman cuprates and structural interconversion}

By employing a Gilman cuprate base, that is of the type $\mathrm{R}_{2} \mathrm{CuLi}$, Gais and co-workers noted in 2008 the attack of $(Z)-$ and $(E)$-alkenyl sulfoximines at the $\alpha$-position and the 1,2metallate rearrangement of the resulting copper derivatives prior to trapping. A pre-complex between the cuprate base and the sulfoximine group was proposed as a possible explanation for the reaction. ${ }^{55}$ However, with the exception of this study, other examples reported in the literature concerning deprotometallation using cuprate bases have involved aromatic substrates. Moreover, it is noteworthy that these studies have focused on Lipshutz cuprates and, as defined in section 2 of this review, Lipshutz-type cuprates. This emphasis is borne of studies on the Gilman cuprates RCu(TMP)Li $n \mathrm{~S}(\mathrm{R}=\mathrm{Ph}, n=3$, $\mathrm{S}=$ THF 19, $\mathrm{R}=\mathrm{Me}, n=1, \mathrm{~S}=$ TMEDA 20) and their tendency to include or exclude LiCN. Crucially, the same work established that 20 was relatively inactive in DoC. ${ }^{34}$ Thus, $N, N$-diisopropylbenzamide was treated both with pre-isolated 20 and a solution containing 20 and LiCN, prior to electrophilic quenching with $\mathrm{I}_{2}$. Results showed that iodination proceeded in both cases, but yielding only $37 \% 2$-iodo- $N, N$-diisopropyl- benzamide when employing pre-isolated 20 . This improved to $89 \%$ using the solution containing 20 and LiCN. Clearly, these data pointed to the importance of the presence of a Lipshutz or Lipshutz-type formulation base in solution, albeit the relationship between Gilman cuprate and $\operatorname{LiX}(\mathrm{X}=\mathrm{CN}$, halide $)$ remained complex.

\subsection{Lipshutz formulations: efficient DoC reagents}

The ability of several lithium cuprate bases to deprotonate aromatic compounds was first evidenced in 2007 by Usui et al. ${ }^{6}$ The efficiency of the bases was evaluated by reacting benzonitrile with a variety of amidocuprates (formally 2 equiv. of cuprate monomer wrt benzonitrile, the cuprate being prepared in situ by the treatment of amidolithium, organolithium and copper cyanide in appropriate ratios) at $0{ }^{\circ} \mathrm{C}$ in THF. This was followed by iodolysis after stirring for $3 \mathrm{~h}$ at room temperature (in Table 1 the cuprates are expressed formally as monomers). Whereas amidocuprates prepared from $\mathrm{CuI}$ failed to give the 2 -iodo derivative in satisfying yields (see next section), it was clear that cuprates of Lipshutz formulation promoted DoC of the aromatic in generally high yields (70-93\%). In particular, the TMP ligand, one of the bulkiest amide ligands available, was identified as being crucial for both good reactivity and chemoselectivity.

The putative cuprate $\mathrm{MeCu}(\mathrm{TMP})(\mathrm{CN}) \mathrm{Li}_{2}$, identified in Table 1 as a very efficient lithium cuprate base for the deprotonation-iodolysis of benzonitrile, was next employed in the attempted functionalization of a variety of other aromatic substrates (Table 2). In this way, the reaction was extended with success to the elaboration of $\mathrm{N}, \mathrm{N}$-diisopropylbenzamide, 3-cyanoanisole, 1,3-dimethoxybenzene and 1,3-dicyanobenzene. Notably, the presence of a bromo or iodo group on the benzene ring was tolerated provided that a lower reaction temperature was used $\left(-78{ }^{\circ} \mathrm{C}, c f .0^{\circ} \mathrm{C}\right.$ in Table 1$)$. Lastly, fivemembered aromatic heterocycles could be similarly regioselectively converted at 0 or $-40^{\circ} \mathrm{C}$.

Table 1 Screening of amidocuprates for benzonitrile-directed ortho cupration

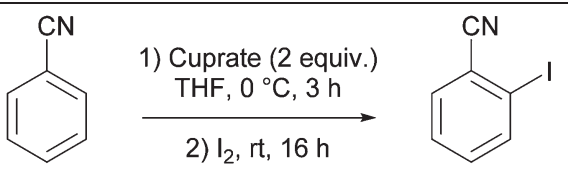

\begin{tabular}{llc}
\hline Entry & Cuprate & Yield $^{a}(\%)$ \\
\hline 1 & TMPCu(CN)Li & 0 \\
2 & $(\mathrm{TMP})_{2} \mathrm{Cu}(\mathrm{I}) \mathrm{Li}_{2}$ & 0 \\
3 & $\mathrm{MeCu}(\mathrm{TMP})(\mathrm{I}) \mathrm{Li}_{2}$ & 51 \\
4 & $\mathrm{MeCu}\left(\mathrm{NMe}_{2}\right)(\mathrm{CN}) \mathrm{Li}_{2}$ & 0 \\
5 & $\mathrm{MeCu}\left(\mathrm{NPr}^{2}\right)(\mathrm{CN}) \mathrm{Li}_{2}$ & 53 \\
6 & $(\mathrm{TMP})_{2} \mathrm{Cu}(\mathrm{CN}) \mathrm{Li}_{2}$ & 74 \\
7 & $\mathrm{MeCu}(\mathrm{TMP})(\mathrm{CN}) \mathrm{Li}_{2}$ & 91 \\
8 & $\mathrm{Bu}^{n} \mathrm{Cu}(\mathrm{TMP})(\mathrm{CN}) \mathrm{Li}_{2}$ & 83 \\
9 & $\mathrm{Bu}^{t} \mathrm{Cu}(\mathrm{TMP})(\mathrm{CN}) \mathrm{Li}_{2}$ & 70 \\
10 & ${\mathrm{PhCu}(\mathrm{TMP})(\mathrm{CN}) \mathrm{Li}_{2}}$ & 93
\end{tabular}

${ }^{a}$ After purification. 
Table 2 DoC of aromatic substrates using $\mathrm{MeCu}(\mathrm{TMP})(\mathrm{CN}) \mathrm{Li}_{2}$

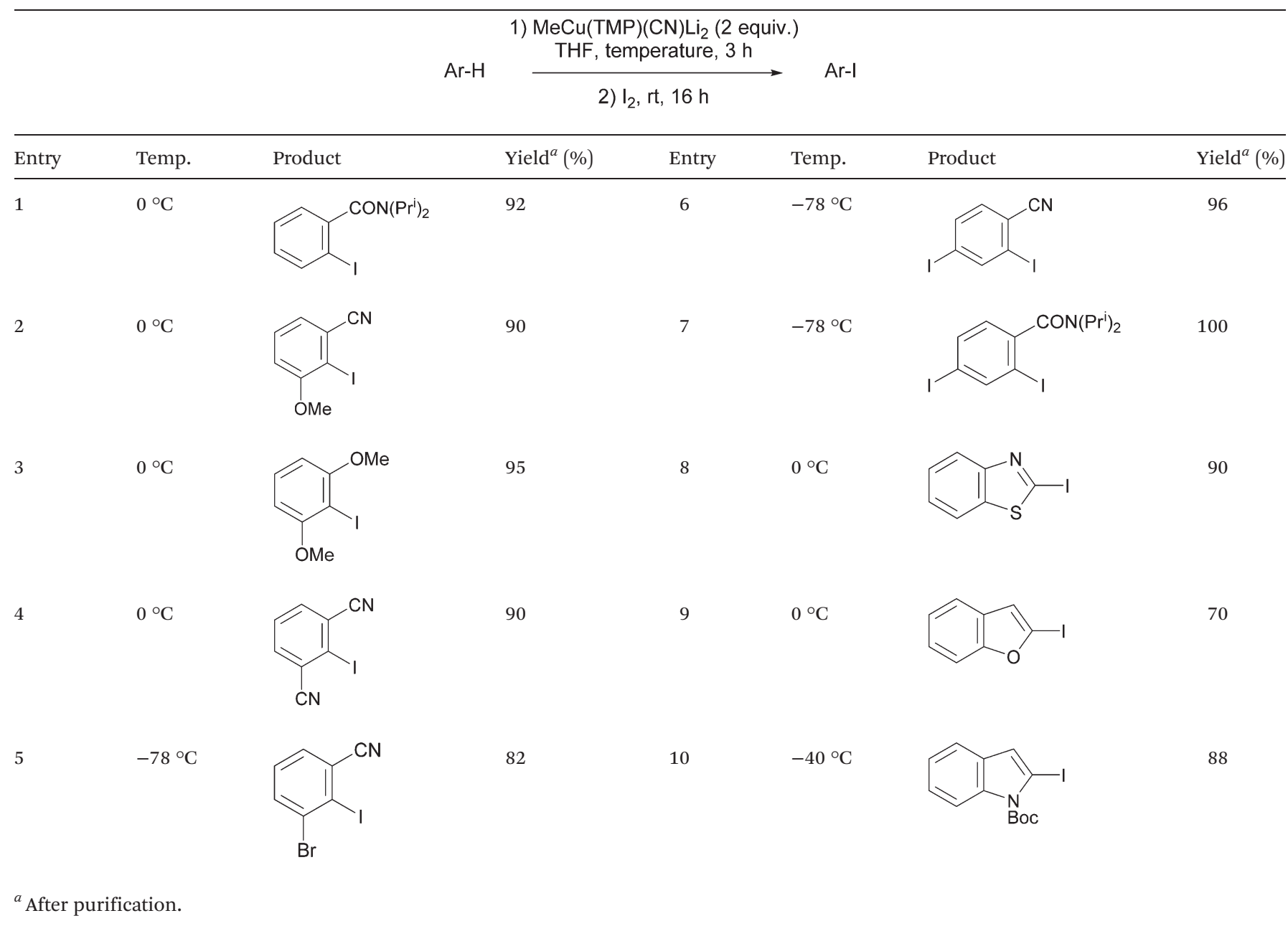

Besides iodine, electrophiles such as $\mathrm{D}_{2} \mathrm{O}$, methyl iodide, allyl bromide, benzoyl chloride and chlorotrimethylsilane were all successfully used to efficiently intercept the arylcuprate intermediate generated by ortho deprotometallating $N, N$-diisopropylbenzamide. These electrophiles afforded the expected deuterated, methylated, allylated, benzoylated and silylated derivatives in yields ranging from 84 to $100 \%$ (Table 3 ). The introduction of a hydroxyl group was achieved in $56 \%$ yield by exposure of the cuprate to molecular oxygen at room temperature in the presence of $\mathrm{CuCN}$ (1 equiv.).

When the arylcuprates prepared from $N, N$-diisopropylbenzamide using different putative $\mathrm{RCu}(\mathrm{TMP})(\mathrm{CN}) \mathrm{Li}_{2}$ bases were treated with nitrobenzene, selective oxidative ligand coupling took place to furnish either the 2-methylated $(\mathrm{R}=\mathrm{Me})$ or the 2-phenylated $(\mathrm{R}=\mathrm{Ph})$ cross-coupled derivative, or even the homocoupled product $(\mathrm{R}=$ TMP) (Scheme 10).

\subsection{Replacing cyanide with halide: Lipshutz-type cuprates}

Since 2009, Mongin and co-workers have extensively documented the use of a cuprate of (TMP) ${ }_{2} \mathrm{Cu}(\mathrm{Cl}) \mathrm{Li}_{2}$ formulation, which has been structurally characterised as both a $\mathrm{THF}^{29}$ and a diethyl ether ${ }^{53}$ solvate (dimers of $\mathbf{9}$ and 14, respectively). In
Table 3 Electrophilic trapping of the arylcuprate generated from $\mathrm{N}, \mathrm{N}$ diisopropylbenzamide

\begin{tabular}{|c|c|c|c|}
\hline & & $\begin{array}{c}\text { 1) } \mathrm{MeCu}(\mathrm{TMP})(\mathrm{CN}) \mathrm{Li}_{2} \\
(2 \text { equiv.) } \\
\underset{\mathrm{THF}, 0^{\circ} \mathrm{C}, 3 \mathrm{~h}}{\stackrel{\text { 2) Electrophile, }}{\longrightarrow}} \\
\text { conditions }\end{array}$ & \\
\hline Entry & \multicolumn{2}{|c|}{ Electrophile, conditions (E) } & Yield $^{a}(\%)$ \\
\hline 1 & \multicolumn{2}{|c|}{$\mathrm{D}_{2} \mathrm{O}, \mathrm{rt}, 0.5 \mathrm{~h}(\mathrm{D})$} & 100 \\
\hline 2 & \multicolumn{2}{|c|}{ MeI, rt, 16 h (Me) } & 99 \\
\hline 3 & \multicolumn{2}{|c|}{ AllylBr, rt, 16 h (Allyl) } & 99 \\
\hline 4 & \multicolumn{2}{|c|}{$\mathrm{PhCOCl}, 80^{\circ} \mathrm{C}, 16 \mathrm{~h}(\mathrm{COPh})$} & 84 \\
\hline 5 & \multicolumn{2}{|c|}{ TMSCl, $50^{\circ} \mathrm{C}, 16 \mathrm{~h}(\mathrm{TMS})$} & 99 \\
\hline
\end{tabular}

synthetic studies, the chloride-containing cuprate was generated either from air-stable $\mathrm{CuCl}_{2}$.TMEDA by reduction of $\mathrm{Cu}^{\text {II }}$ to $\mathrm{Cu}^{\mathrm{I}}$ using butyllithium (1 equiv.) followed by the addition of TMPLi (2 equiv.) $)^{56}$ or, more simply, from $\mathrm{CuCl}$ by the addition of TMPLi (2 equiv.). ${ }^{57}$ In spite of the clear structural similarities between the chloride- $(\mathbf{9} / \mathbf{1 4})$ and iodide-containing 

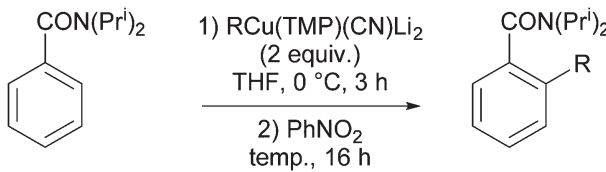

$\mathrm{R}=\operatorname{Me}\left(0^{\circ} \mathrm{C}\right): 93 \%$
$\mathrm{R}=\operatorname{Ph}(\mathrm{rt}): 91 \%$

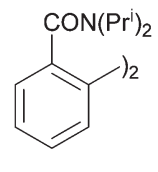

$\mathrm{R}=\mathrm{TMP}\left(0^{\circ} \mathrm{C}\right):$ $100 \%$
Scheme 10 Oxidation of arylcuprates coming from $N, N$-diisopropylbenzamide.

(12/16) lithium cuprates, it is clear that the (TMP) ${ }_{2} \mathrm{Cu}(\mathrm{Cl}) \mathrm{Li}_{2}$ formulation base prepared in situ behaves significantly differently to the (TMP) ${ }_{2} \mathrm{Cu}(\mathrm{I}) \mathrm{Li}_{2}{ }^{32}$ formulation base in these systems and, instead, acts rather more like the Lipshutz formulation cuprate (TMP) ${ }_{2} \mathrm{Cu}(\mathrm{CN}) \mathrm{Li}_{2}$ (see above) ${ }^{6}$ inasmuch as it is capable of deprotonating a large range of aromatic substrates. Similar results were achieved recently utilising the bromidecontaining Lipshutz-type system (viz. 10 and $\mathbf{1 5}$ above) though with less impressive conversions than were typically attainable with the chloride. ${ }^{30}$ Taken together, these data are consistent with the view, expressed in the structural discussion above, that there is no fundamental change incurred to the cuprate on replacing $\mathrm{X}=\mathrm{CN}$ (Lipshutz formulation) with $\mathrm{X}=$ halide (Lipshutz-type formulation) but rather, as the halide gets softer there is a general trend away from the reactivity patterns seen for the Lipshutz system. Overall, therefore, while structural evidence so far suggests the adherence of both Lipshutz and Lipshutz-type species to the general formula $\mathrm{R}_{2} \mathrm{Cu}(\mathrm{X}) \mathrm{Li}_{2}$, reactivity studies suggest that as the softness of $\mathrm{X}$ is increased it becomes reasonable to propose a gradual progression in solution to $\mathrm{R}_{2} \mathrm{CuLi} \cdot \mathrm{LiX}$.

Reaction conditions were optimized for the chloride-containing cuprate using 2,4-dimethoxypyridine as substrate. Using THF as solvent, the best yield (60\%) was recorded by carrying out the reaction with the base (1 equiv.) for $2 \mathrm{~h}$ at room temperature before interception with benzoyl chloride. The reaction could be efficiently carried out in toluene containing TMEDA (5 equiv.), but to the detriment of the regioselectivity. Using diethyl ether instead of THF led to the expected ketone in an improved 99\% yield (Scheme 11). Meanwhile, the use of mixed organo/amido lithiocuprates resulted in lower yields due to a reduced ability to effect deprotonation and the occurrence of competitive side reactions.

The cuprate generated regioselectively from 2,4-dimethoxypyrimidine could be converted into ketones using 2- and 4-chlorobenzoyl chlorides. The 5-methyl derivative was synthesized in a reasonable yield using methyl iodide whereas a low $19 \%$ yield was noted for the 5 -allylated derivative (a result that could have been due to the presence of the nucleophilic diazine nitrogen) (Table 4). Using nitrobenzene as an oxidant resulted in formation of the corresponding 5,5'-bipyrimidine in $22 \%$ yield.

Reaction conditions for ketone formation were carefully optimized, taking account of both steps, using 2-methoxypyridine as substrate and benzoyl chloride to intercept the intermediate arylcuprate (Table 5). Under the conditions used
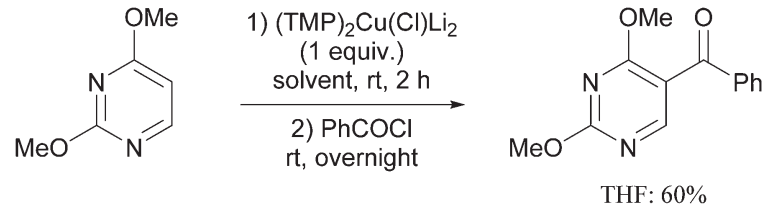

THF: $60 \%$

HexH + TMEDA (5 equiv): $29 \%$ PhMe + TMEDA ( 5 equiv): $63 \%$ ( $6 \%$ of the 6 -substituted derivative) $\mathrm{Et}_{2} \mathrm{O}: 99 \%$

Scheme 11 Deprotocupration-benzoylation of 2,4-dimethoxypyrimidine.

Table 4 Electrophilic trapping of the cuprate coming from 2,4dimethoxypyrimidine

\begin{tabular}{|c|c|c|c|}
\hline $\mathrm{MeO}^{-}$ & OMe & $\begin{array}{c}\text { 1) } \begin{array}{c}(\mathrm{TMP})_{2} \mathrm{Cu}(\mathrm{Cl}) \mathrm{Li}_{2} \\
(1 \text { equiv.) }\end{array} \\
\underset{\mathrm{THF}, \mathrm{rt}, 2 \mathrm{~h}}{\mathrm{2} \text { ) Electrophile, }} \\
\text { temp., overnight }\end{array}$ & Me \\
\hline Entry & \multicolumn{2}{|c|}{ Electrophile (E), temp. } & Yield $^{a}(\%)$ \\
\hline 1 & \multicolumn{2}{|c|}{ 2- $\mathrm{ClC}_{6} \mathrm{H}_{4} \mathrm{COCl}, \mathrm{rt}\left(\mathrm{COC}_{6} \mathrm{H}_{4}-2-\mathrm{Cl}\right)$} & 58 \\
\hline 2 & \multicolumn{2}{|c|}{$4-\mathrm{ClC}_{6} \mathrm{H}_{4} \mathrm{COCl}, 60^{\circ} \mathrm{C}\left(\mathrm{COC}_{6} \mathrm{H}_{4}-4-\mathrm{Cl}\right)$} & 52 \\
\hline 3 & \multicolumn{2}{|c|}{$\mathrm{MeI}, 30^{\circ} \mathrm{C}(\mathrm{Me})$} & 62 \\
\hline 4 & \multicolumn{2}{|c|}{ AllylBr, rt (Allyl) } & 19 \\
\hline
\end{tabular}

above for reaction of 2,4-dimethoxypyrimidine, the expected 3 -benzoylpyridine was isolated in $82 \%$ yield. By using 0.5 equiv. of cuprate base, the yield was reduced by half, while reducing or extending the deprotonation time was deleterious for the yield.

Interestingly, the temperature at which the quenching step was conducted in the deprotocupration-benzoylation of 2-methoxypyridine was found to have an impact on the course of

Table 5 Deprotocupration-benzoylation of 2-methoxypyridine

\begin{tabular}{|c|c|c|c|c|c|c|}
\hline & & $\mathrm{OMe}$ & \multicolumn{2}{|c|}{ 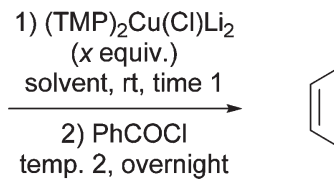 } & Me & \\
\hline Entry & $x$ & Solvent & & Time 1 & Temp. 2 & $\begin{array}{l}\text { Yield }^{a} \\
(\%)\end{array}$ \\
\hline 1 & 1 & $\mathrm{THF}$ & & $2 \mathrm{~h}$ & $\mathrm{rt}$ & 82 \\
\hline 2 & 0.5 & THF & & $2 \mathrm{~h}$ & $\mathrm{rt}$ & 40 \\
\hline 3 & 1 & THF & & $0.5 \mathrm{~h}$ & $\mathrm{rt}$ & 72 \\
\hline 4 & 1 & THF & & $6 \mathrm{~h}$ & $\mathrm{rt}$ & 62 \\
\hline 5 & 1 & THF & & $2 \mathrm{~h}$ & $60^{\circ} \mathrm{C}$ & $66^{b}$ \\
\hline 6 & 1 & $\mathrm{THF}$ & & $2 \mathrm{~h}$ & $0^{\circ} \mathrm{C}$ & 66 \\
\hline 7 & 1 & $\mathrm{Et}_{2} \mathrm{O}$ & & $2 \mathrm{~h}$ & $\mathrm{rt}$ & 71 \\
\hline 8 & 1 & PhMe + & TMEDA (5 equiv.) & $2 \mathrm{~h}$ & $\mathrm{rt}$ & 49 \\
\hline 9 & 1 & $\mathrm{Et}_{2} \mathrm{O}+{ }^{\prime}$ & MEDA (5 equiv.) & $2 \mathrm{~h}$ & $\mathrm{rt}$ & 81 \\
\hline
\end{tabular}

${ }^{a}$ After purification. ${ }^{b}$ The 3,6-disubstituted derivative was also formed in $10 \%$ yield. 
the reaction. Hence, when trapping was performed at $60{ }^{\circ} \mathrm{C}$ the yield decreased on account of the competitive degradation (as observed for extended deprotonation times at room temperature). In contrast, at $0{ }^{\circ} \mathrm{C}$ the reaction proved slower. In the case of 2-methoxypyridine, switching solvent medium from THF to diethyl ether or toluene containing TMEDA did not improve the yield. A good yield was restored by using diethyl ether containing dimethyl sulfide ( 5 equiv.), a cosolvent which has been proven to be useful in deprotocupration reactions. ${ }^{58}$

Besides aroyl chlorides, other electrophiles were also evaluated for their room temperature-reactivity towards the pyridylcuprate intermediate (Table 6). Employing 2-phenyloxirane led to both regioisomeric alcohols. Meanwhile, the use of diphenyl disulfide afforded the expected functionalized pyridine more efficiently. Reactions involving conjugate addition were only found to be possible using alkyl propionates, affording the products as $(Z)$ and $(E)$ mixtures in only moderate yields. By using iodine, the expected 3-iodo derivative was formed, but again in a moderate (28\%) yield on account of competitive homocoupling taking place. 4-Chlorobenzenesulfonyl chloride and, to a greater extent, dimethylsulfamoyl chloride and tetramethylthiuram behaved as oxidative agents, furnishing the 3,3'-dimer. From 2-fluoropyridine, 3,3'-dimer formation was similarly noted using chlorotrimethylsilane at $50{ }^{\circ} \mathrm{C}(52 \%$ yield), chloranil at $60{ }^{\circ} \mathrm{C}(94 \%)$ and nitrobenzene at room temperature (84\%).

Table 6 Electrophilic trapping of the cuprate generated from 2-methoxypyridine

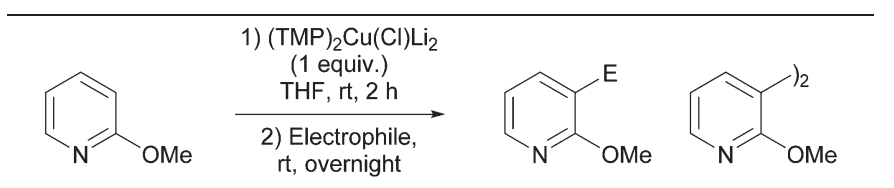

\begin{tabular}{llll}
\hline Entry & Electrophile (E) & Yield(s) ${ }^{a}(\%)$ & \\
\hline $1^{b}$ & $2-\mathrm{ClC}_{6} \mathrm{H}_{4} \mathrm{COCl}\left(\mathrm{COC}_{6} \mathrm{H}_{4}-2-\mathrm{Cl}\right)$ & 56 & - \\
$2^{b}$ & $2-\mathrm{BrC}_{6} \mathrm{H}_{4} \mathrm{COCl}\left(\mathrm{COC}_{6} \mathrm{H}_{4}-2-\mathrm{Br}\right)$ & 43 & - \\
$3^{b}$ & & &
\end{tabular}

4

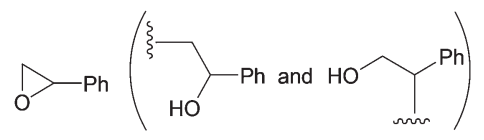

22 and 12

67

$39(Z)$ and $10^{c}(E)$

$26(E)$

$30(Z)$ and $17(E)$

28

3

-

$-$
That azines and diazines can be functionalized through deprotonative metallation at room temperature makes the method described above of interest. Indeed, such $\pi$-deficient heterocycles are sensitive to nucleophilic attacks on their $\mathrm{C}=\mathrm{N}$ bond due to their low LUMO levels. As a consequence, traditional lithium bases, when they can be employed, in general require very low temperature reaction conditions. ${ }^{59}$ The optimized deprotocupration-aroylation conditions were thus applied to the synthesis of different azinic and diazinic ketones (Table 7). ${ }^{28-30,56,57}$ Good results were observed using 2substituted pyridine substrates (with the exception of 2-bromopyridine, from which elimination of metal halide to give 2,3pyridine was suspected), ${ }^{60}$ with TMEDA favouring the reaction and regioselectivities being analogous to those observed using TMPLi. ${ }^{59}$ Except for 4-methoxypyridine and 3,5-dichloropyridine, the reaction required the presence of a substituent at the position adjacent to the ring nitrogen.

In the framework of recent studies, the behaviour of 1,4dimethoxybenzene and five-membered aromatic heterocycles towards $(\mathrm{TMP})_{2} \mathrm{Cu}(\mathrm{Cl}) \mathrm{Li}_{2}$ was also investigated (Table 8). Under the reaction conditions used for the functionalization of pyridines described above, 1,4-dimethoxybenzene was converted to its 2-allylated derivative and to diarylketones in medium yields. No improvement was noted by increasing the deprotonation temperature to $40^{\circ} \mathrm{C}$.

Continuing in the same vein, $N$-Boc indole, furan and thiophene were similarly reacted in the deprotocupration-aroylation sequence to afford the expected ketones in approximately $50 \%$ yield. In order to reach higher yields, THF had to be replaced by either diethyl ether or diethyl ether containing dimethyl sulfide ( 5 equiv.). As expected, when this was undertaken, higher conversions were noted, although mixtures of 2substituted and 2,5-disubstituted thiophenes also formed. From ethyl 2-thiophenecarboxylate and 3-thiophenecarboxylate, the reactions took place at the 5 and 2 position of the aromatic ring respectively, but with a lower chemoselectivity in the latter case. Finally, it was noted that the trapping temperature had an important impact on the efficiency on the reaction, with the best yields being obtained at $60^{\circ} \mathrm{C}$ (Table 9).

Whereas the arylcuprates prepared from $N, N$-diisopropylbenzamide using different $\mathrm{RCu}(\mathrm{TMP})(\mathrm{CN}) \mathrm{Li}_{2}$ bases were converted to the 2-methylated $(\mathrm{R}=\mathrm{Me})$ or the 2 -phenylated $(\mathrm{R}=$ $\mathrm{Ph}$ ) cross-coupled derivative upon treatment by nitrobenzene (Scheme 10), ${ }^{6}$ the arylcuprates obtained by deprotocupration using (TMP) $)_{2} \mathrm{Cu}(\mathrm{Cl}) \mathrm{Li}_{2}$ could be utilised in palladium-catalysed cross-couplings with aryl halides such as 4-iodoanisole and 2-bromopyridine in the presence of catalytic amounts of $\mathrm{PdCl}_{2}\left(\mathrm{PPh}_{3}\right)_{2}$ and dppf (1,1'-bis(diphenylphosphino)ferrocene) (Table 10). ${ }^{57}$

Compared with the reactivities of previously reported lithium-zinc bases, it is clear that the lithiocuprate $(\mathrm{TMP})_{2} \mathrm{Cu}(\mathrm{Cl}) \mathrm{Li}_{2}$ behaves more like a lithium amide, as it leads to deprotonation of the most acidic hydrogen atoms and is incompatible with reactive functional groups. This, however, is offset by a satisfying reactivity towards a larger range of electrophiles. 
Table 7 Deprotocupration-aroylation of azine and diazine substrates

$$
\begin{aligned}
& \begin{array}{l}
\text { 1) }(\mathrm{TMP})_{2} \mathrm{Cu}(\mathrm{Cl}) \mathrm{Li}_{2} \\
\text { (1 equiv.) }
\end{array} \\
& \text { Ar-H } \underset{\substack{\text { 2) } \mathrm{ClCOAr} \\
\text { it, overnight }}}{\text { THF, rt, } 2 \mathrm{~h}} \quad \text { Ar-COAr' }
\end{aligned}
$$

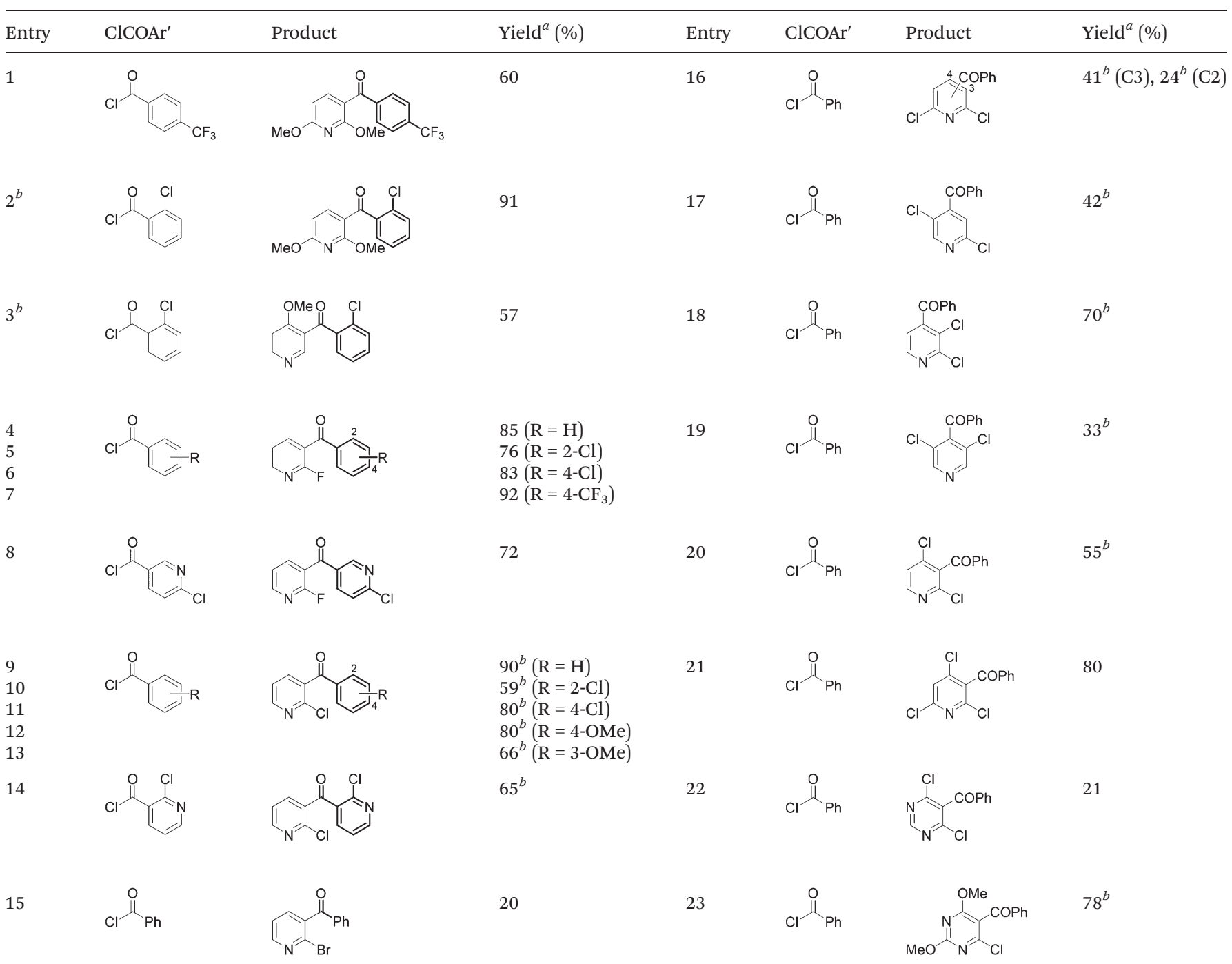

${ }^{a}$ After purification. ${ }^{b}$ Base prepared in the presence of TMEDA (1 equiv.).

\subsection{New amidocuprate adducts and the formation of reactive species}

Lastly, and in line with the investigation of less expensive amide ligands, ${ }^{51}$ the effect of replacing TMP with DMP has been studied by Harford et al. ${ }^{53}$ As noted above, attempts to fabricate Lipshutz-type cuprates analogous to 9, 10, 12 and 14-16 instead afforded the series of complexes 28, 29, 31 and 32 that have been interpreted as adducts between Lipshutztype and Gilman cuprates. The potential importance of this new structure-type is explained fully in the final section of this review where DFT analysis has suggested that the abstraction of LiX from Lipshutz or Lipshutz-type cuprates is fundamental to incurring in situ formation of the reactive base in DoC. It is clear, therefore, that these new adducts can all be viewed in terms of the elimination of 1 equiv. Lix having occurred from the corresponding Lipshutz-type dimer (e.g. $\left.\mathbf{1 4}_{2} \rightarrow \mathbf{2 8}+\mathrm{LiCl}\right)$. Crucial to this view of their importance, using the base prepared in situ from DMPLi (4 equiv.) and CuBr (2 equiv.) in THF, $N, N$-diisopropylbenzamide was readily converted to the corresponding 2-metallated derivative, as shown by subsequent trapping with iodine ( $80 \%$ yield). An essentially 
Table 8 Electrophilic trapping of the cuprate coming from 1,4dimethoxybenzene

Electrophile (E)

identical result (82\% yield) was recorded using pre-isolated $\left[(\mathrm{DMP})_{2} \mathrm{CuLi}\left(\mathrm{OEt}_{2}\right)\right]_{2} \mathrm{LiBr} 28$ (1 equiv.). It is clear, therefore, that efficient DoC is achievable not only by applying bases of Lipshutz(-type) formulation but also by using newly reported adducts.

\section{DFT modelling of cuprate structure and DoC reaction pathways}

As noted in section 2 of this review, there has been controversy in the literature for some time regarding the behaviour of cyanide ligands in so-called Lipshutz cuprates. Spectroscopic data were used in 1990 to support both the view that the structure might be lower-order (with no copper-cyanide interaction $)^{18}$ and that it might be higher-order (Cu-CN bonded). ${ }^{19}$ This led to theoretical studies on a variety of solvated lithium dialkylcuprates of formulation $\mathrm{Me}_{2} \mathrm{Cu}(\mathrm{CN}) \mathrm{Li}_{2} \cdot 2 \mathrm{OH}_{2} \cdot{ }^{20,22,61}$

Table 9 Deprotocupration-aroylation of five-membered aromatic heterocycles

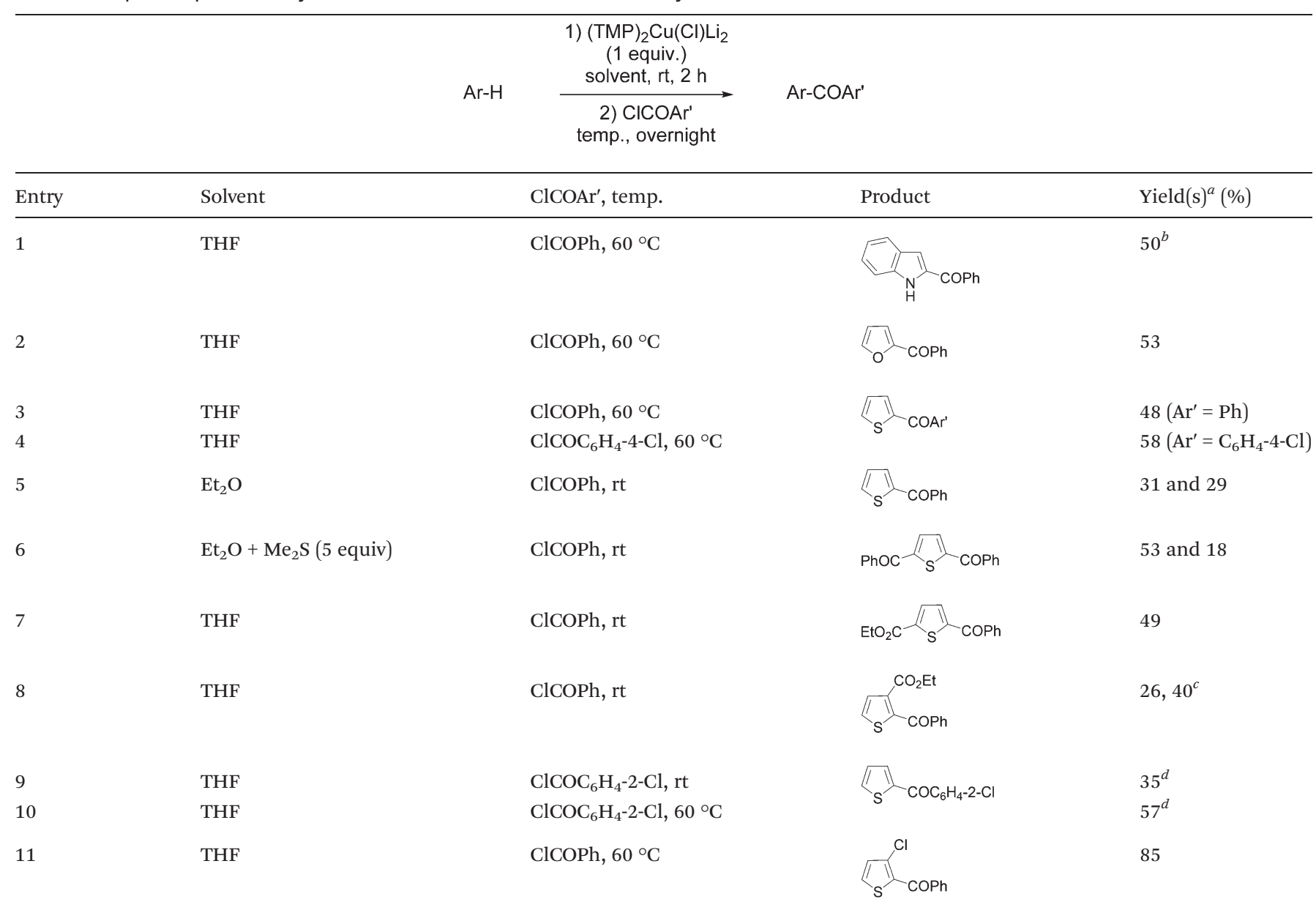

${ }^{a}$ After purification. ${ }^{b}$ After removal of the tert-butoxycarbonyl (Boc) group. ${ }^{c}$ Metallation step performed at $0{ }^{\circ} \mathrm{C} .{ }^{d}$ Base prepared in the presence of TMEDA (1 equiv.). 
Table 10 Palladium-catalysed cross-coupling of arylcuprates

$$
\text { Ar-H } \quad \begin{gathered}
\text { 1) }(\mathrm{TMP})_{2} \mathrm{Cu}(\mathrm{Cl}) \mathrm{Li}_{2} \\
(0.5 \text { equiv. }
\end{gathered} \quad \underset{\mathrm{THF}, \mathrm{rt}, 2 \mathrm{~h}}{2) \mathrm{XAr}^{\prime}} \quad \mathrm{Ar}-\mathrm{Ar}^{\prime}
$$

\section{Ar-Ar'}

Product<smiles>[R]c1ccc(-c2cccnc2OC)cc1</smiles><smiles>COc1[Y]cc(-c2ccc(OC)cc2)c(OC)c1</smiles>

7<smiles>COc1ccc(I)cc1</smiles><smiles>COc1ccc(-c2cc(OC)ccc2OC)cc1</smiles>

8<smiles>Brc1ccccn1</smiles><smiles>[R]c1ccc(-c2ccccn2)c(OC)n1</smiles><smiles>COc1ncc(-c2ccccn2)c(OC)n1</smiles><smiles>Brc1ccccn1</smiles>

11<smiles>Brc1ccccn1</smiles>

44

Yield $^{a}(\%)$

$64(\mathrm{X}=\mathrm{I}, \mathrm{R}=\mathrm{OMe})$ $46(\mathrm{X}=\mathrm{Br}, \mathrm{R}=\mathrm{OMe})$ $47(\mathrm{X}=\mathrm{Br}, \mathrm{R}=\mathrm{F})$ $64(\mathrm{X}=\mathrm{Br}$, $\mathrm{R}=\mathrm{CO}_{2} \mathrm{Et}$

$51(\mathrm{Y}=\mathrm{CH}, \mathrm{Z}=\mathrm{N})$ $42,46^{b}(\mathrm{Y}=\mathrm{Z}=\mathrm{N})$

$9^{b}$$$
63(\mathrm{R}=\mathrm{H})
$$
$54(\mathrm{R}=\mathrm{OMe})$

$36^{b}$

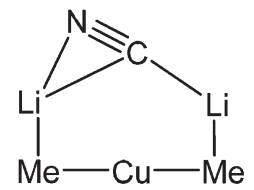

Fig. 19 Preferred ab initio structure of alkylcuprate $\mathrm{Me}_{2} \mathrm{Cu}(\mathrm{CN}) \mathrm{Li}_{2}$.
More recently, among the organocuprate(I) complexes receiving increased attention have been heteroleptic cuprates $\left(\left[\mathrm{R}-\mathrm{Cu}-\mathrm{R}^{\prime}\right]^{-}\right)$. Organyl(amido)cuprates represent an important class of these, particularly since they have now been harnessed to achieve a variety of organic transformations. Thus, 2007 saw publication of the first of several reports that TMP-cuprates of the type $\mathrm{RCu}(\mathrm{TMP})(\mathrm{CN}) \mathrm{Li}_{2}(\mathrm{R}=$ alkyl, phenyl, TMP) could promote the highly chemoselective DoC of multifunctionalized aromatic compounds. ${ }^{6,34,57}$ The arylcuprate intermediates presumed to result from deprotometallation of the aromatic substrate were effectively employed not only in the trapping of electrophiles, but also in oxidative ligand coupling to form new C-C bonds with alkyl/aryl groups (see section 3.2).

The chemistry of organocuprates(I) has traditionally been dominated by two structure types: Gilman cuprates and Lipshutz(-type) cuprates. As revealed above, these have now been structurally elucidated, with the former capable of exhibiting a homodimeric structure and Lipshutz-type cuprates demonstrating heteroaggregate structures based on metallocycles that are either 6-membered (halide-containing) or 7-membered (cyanide-containing, Scheme 12). Overall, with the help of $\mathrm{X}$-ray crystallography, structure-reactivity relationship studies of TMP-cuprates have revealed that (1) the cyclic dimer of Gilman cuprate (TMP) $)_{2} \mathrm{CuLi}$ is unreactive in DoC and (2) a variety of Lipshutz-type amidocuprates, including (TMP) ${ }_{2} \mathrm{Cu}(\mathrm{CN}) \mathrm{Li}_{2}$, exhibit enhanced deprotonative reactivity. ${ }^{32}$

Further insights into the natures of active cuprate species and the mechanisms by which they exhibit $\mathrm{DoC}$ have been gained through DFT study of the derivatisation of $N, N$-dimethylbenzamide. The deprotonation process using $\mathrm{MeCu}\left(\mathrm{NMe}_{2}\right)(\mathrm{CN}) \mathrm{Li}_{2} \cdot 2 \mathrm{OMe}_{2}$ as a model Lipshutz cuprate is summarized in Scheme $13 .{ }^{32}$

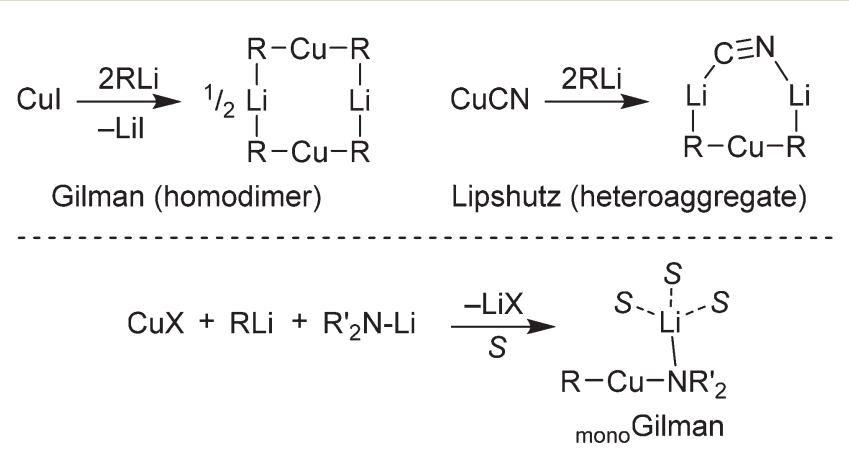

Scheme 12 Structures of Gilman and Lipshutz-type cuprates (top) and monoGilman-type cuprates with solvent coordination (bottom, $S=$ solvent molecule). 

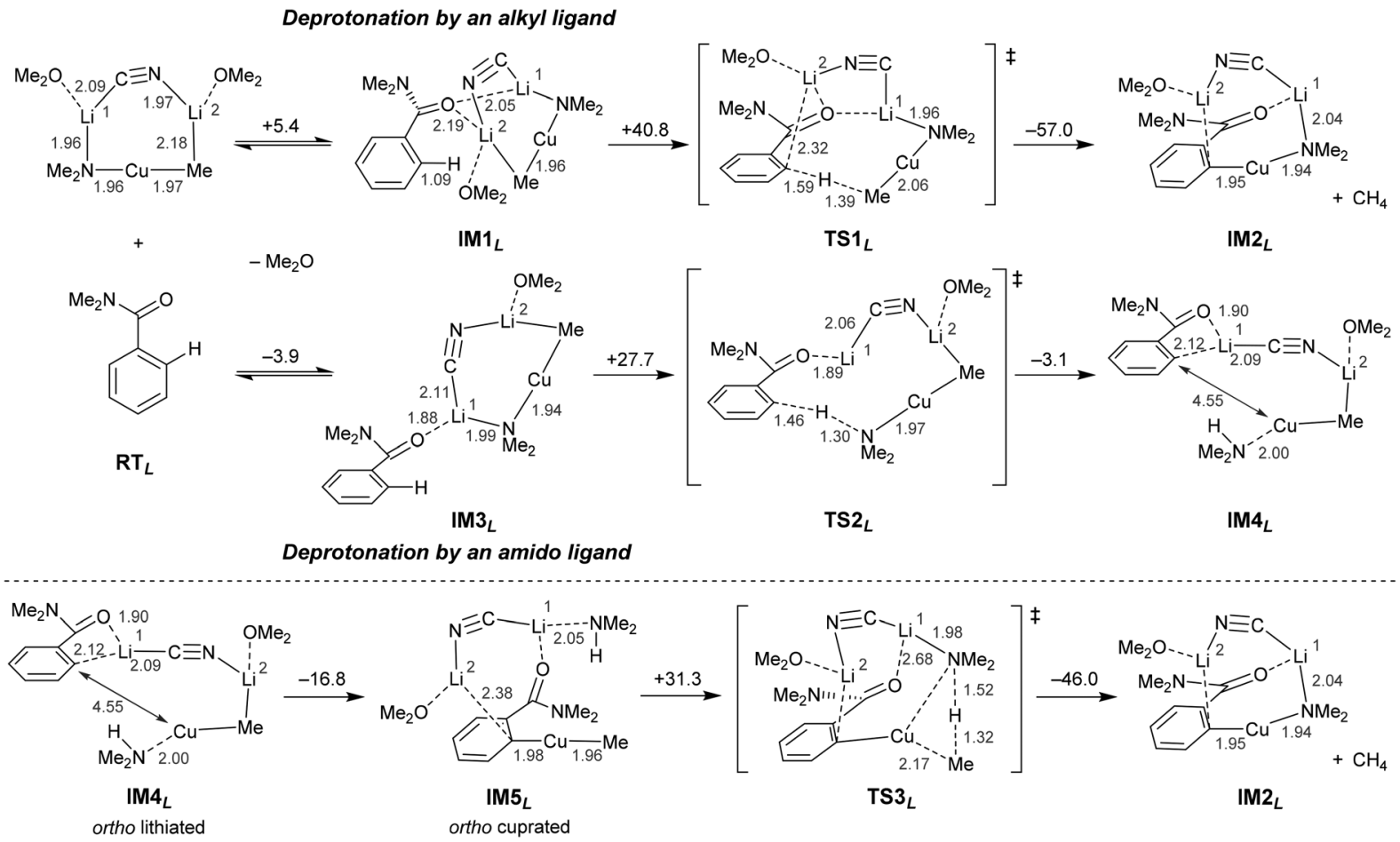

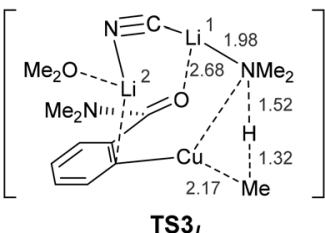

TS3,

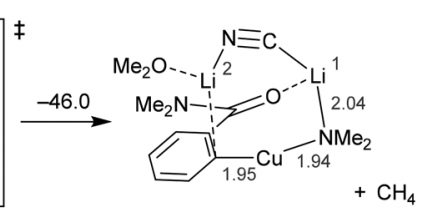

IM2 $L_{L}$

Ligand exchange process - Deprotonation of $\mathrm{Me}_{2} \mathrm{NH}$ by an alkyl ligand

Scheme 13 Proposed reaction pathways for the DoC of $\mathrm{N}, \mathrm{N}$-dimethylbenzamide using Lipshutz cuprate $\mathrm{MeCu}\left(\mathrm{NMe}_{2}\right)(\mathrm{CN}) \mathrm{Li}_{2} \cdot 2 \mathrm{OMe}$. Energy changes and bond lengths at the B3LYP/6-31+G* and SVP $(\mathrm{Cu})$ level of theory are shown in $\mathrm{kcal}^{\mathrm{mol}}{ }^{-1}$ and $\AA$, respectively.

In common with the reaction pathways previously proposed for TMP-zincates ${ }^{2,47}$ and TMP-aluminates, ${ }^{46}$ two plausible pathways were discovered for regioselective DoM (Directed ortho Metallation). These differ in terms of whether the deprotonation step is triggered by the amido or the alkyl ligand in the cuprate. Although in Scheme 13 the formation of $\mathbf{I M} 2_{L}$ represents a thermodynamically favorable outcome, the pathway by which the alkyl ligand promotes the ortho deprotonation $\left(\mathbf{I M 1} 1_{L}-\mathbf{T S} \mathbf{1}_{\boldsymbol{L}}-\mathbf{I M} \mathbf{2}_{\boldsymbol{L}}\right)$ is kinetically unfavorable on account of there being a relatively high energy transition state $\left(\mathbf{T S 1}_{L}, \Delta G^{\ddagger}\right.$ $=40.8 \mathrm{kcal} \mathrm{mol}^{-1}$ ). In contrast, $\mathbf{T S} 2_{\boldsymbol{L}}$ represents the transition state immediately preceding amido ligand-promoted deprotonation $\left(\mathbf{I M} 3_{\boldsymbol{L}}-\mathbf{T S} 2_{\boldsymbol{L}}-\mathbf{I M} \mathbf{4}_{\boldsymbol{L}}\right)$. While the activation barrier to $\mathbf{T S} 2_{\boldsymbol{L}}$ formation is somewhat high $\left(+27.7 \mathrm{kcal} \mathrm{mol}^{-1}\right)$ relative to reaction pre-complex $\mathbf{I M} 3_{L}$, it is still reasonably accessible and can be viewed as allowing metalated intermediate $\mathbf{I M} \mathbf{4}_{\boldsymbol{L}}$ to form. The ligand exchange step (IM5 $\left.\mathbf{S}_{\boldsymbol{L}}-\mathbf{T S} \mathbf{3}_{\boldsymbol{L}}-\mathbf{I M} \mathbf{2}_{\boldsymbol{L}}\right)$, a process seen in TMP-zincate chemistry ${ }^{47}$ but not in TMP-aluminate chemistry, ${ }^{46}$ has a higher activation barrier $\left(+31.3 \mathrm{kcal} \mathrm{mol}^{-1}\right)$. Taken together with the observation of heterocoupling processes achieved using organyl(amido)cuprate reagents (discussed in section 3.2) it is reasonable to conclude that the ligand exchange step would be difficult in DoC.

Interestingly, investigations into possible DoC pathways using the Lipshutz reagent $\mathrm{MeCu}\left(\mathrm{NMe}_{2}\right)(\mathrm{CN}) \mathrm{Li}_{2} \cdot 2 \mathrm{OMe}_{2}$ have led to the identification of another ortho cupration pathway that involves an active monomeric Gilman complex (Scheme 12, bottom). Importantly, the monomeric structure-type of this socalled monoGilman species has been established experimentally using TMP-cuprates isolated from various Lewis basic media. ${ }^{34}$ Calculations that take the Lipshutz reagent $\mathrm{MeCu}\left(\mathrm{NMe}_{2}\right)(\mathrm{CN})$ $\mathrm{Li}_{2} \cdot 2 \mathrm{OMe}_{2}$ as a starting point suggest that the monoGilman complexes $\mathbf{I M} \mathbf{1}_{\boldsymbol{G}}$ and $\mathbf{I M} \mathbf{3}_{\boldsymbol{G}}$ can be formed by the elimination of solvated LiCN (Scheme 14). Although IM1 $\mathbf{I}_{\boldsymbol{G}}$ is more stable than $\mathbf{I M} \mathbf{3}_{G}$, its alkyl ligand fails to promote the deprotonative step in the reaction $\left(\mathbf{I M} \mathbf{1}_{\boldsymbol{G}}-\mathbf{T S} \mathbf{1}_{\boldsymbol{G}}-\mathbf{I M} \mathbf{2}_{\boldsymbol{G}}\right)$, this sequence having a very high activation energy $\left(+40.3 \mathrm{kcal} \mathrm{mol}^{-1}\right)$. In contrast, the pathway $\mathbf{I M} 3_{G^{-}} \mathbf{T S} 2_{G}-\mathbf{I M} \mathbf{4}_{G}$, in which deprotonation is promoted by the amido ligand in the monoGilman base, has a very low activation barrier $\left(+14.9 \mathrm{kcal} \mathrm{mol}{ }^{-1}\right.$ with respect to $\left.\mathbf{I M} \mathbf{3}_{\boldsymbol{G}}\right)$ en route to $\mathbf{I M 4}_{\boldsymbol{G}}$. Although the total activation energy (26.0 kcal mol${ }^{-1}$ ) from $\mathbf{R T}_{\boldsymbol{L}}$ to $\mathbf{T S} \mathbf{2}_{\boldsymbol{G}}$ is comparable to that from $\mathbf{R T}_{\boldsymbol{L}}$ to $\mathbf{T S} 2_{\boldsymbol{L}}\left(23.8 \mathrm{kcal} \mathrm{mol}^{-1}\right.$, in Scheme 13), it is noteworthy that the monoGilman-pathway does not require more than $15 \mathrm{kcal}$ $\mathrm{mol}^{-1}$ for any individual step, and this contrasts with the Lipshutz pathway (which requires more than $27 \mathrm{kcal} \mathrm{mol}^{-1}$ for the deprotonation step $\mathbf{I M} \mathbf{3}_{\boldsymbol{L}}-\mathbf{T S} \mathbf{2}_{\boldsymbol{L}}$ ). As with the Lipshutz system, quenching of the amine $\mathbf{M e}_{2} \mathrm{NH}\left(\mathbf{I M} 5_{G}-\mathbf{T S} \mathbf{3}_{\boldsymbol{G}}-\mathbf{I M} \mathbf{2}_{\boldsymbol{G}}\right)$ appears to be unlikely to proceed on account of the rather high energy difference between $\mathbf{R T}_{\boldsymbol{L}}$ and $\mathbf{T S} \boldsymbol{3}_{\boldsymbol{G}}$ (more than $28 \mathrm{kcal} \mathrm{mol}^{-1}$ ), though the activation barrier to reach $\mathbf{T S}_{\boldsymbol{G}}$ is entropically permissible at $22.4 \mathrm{kcal} \mathrm{mol}^{-1}$.

Oxidative ligand coupling involving the putative arylcuprate intermediate generated from the DoC of $N, N$-diisopropylbenza- 

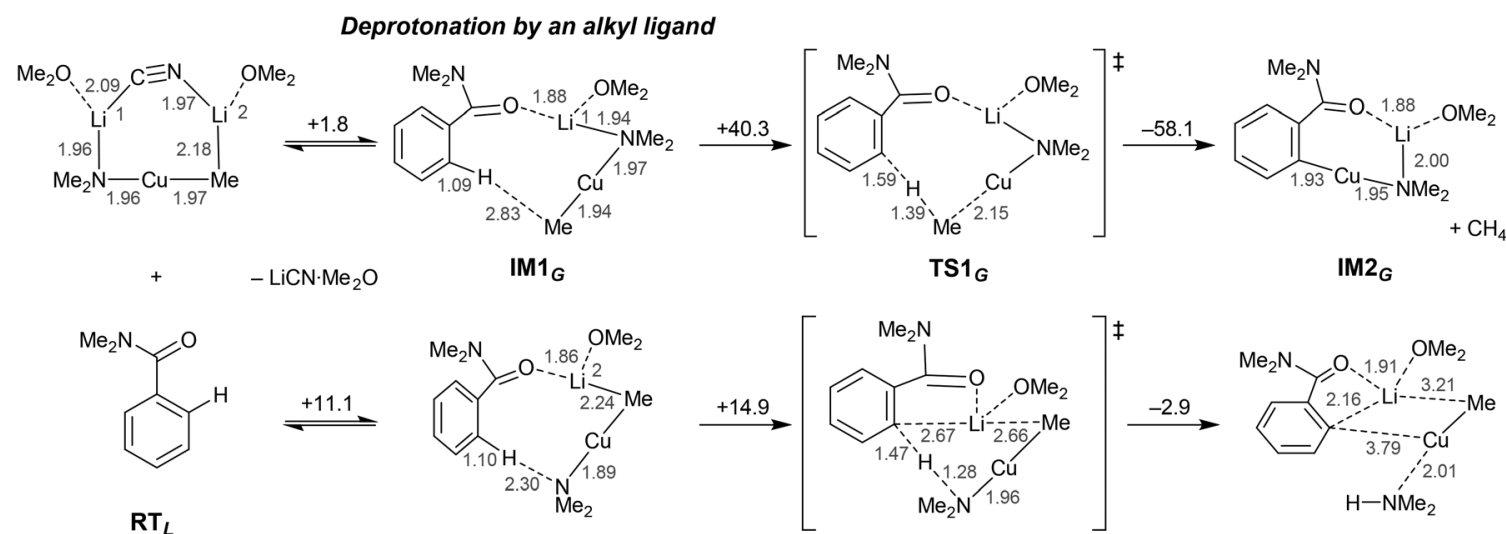

monoGilman Intermediate

$\mathrm{TS2}_{G}$

$1 \mathrm{M} \mathbf{4}_{G}$

Deprotonation by an amido ligand

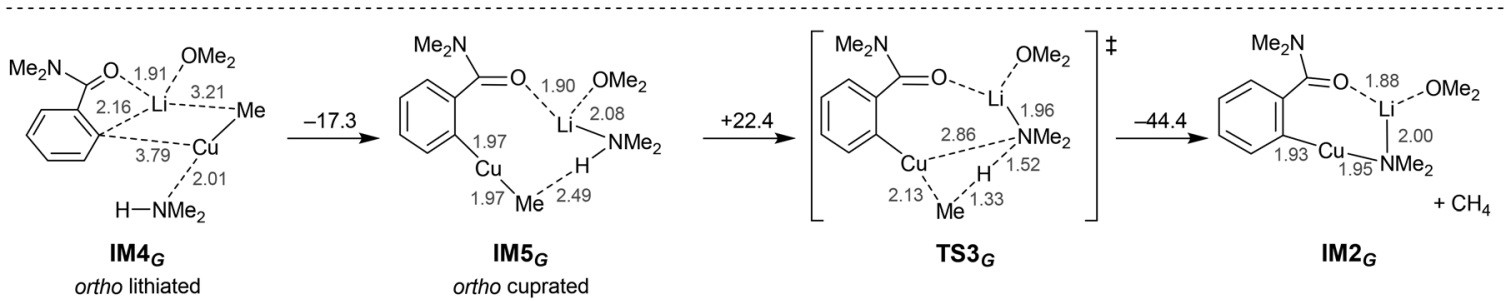

Ligand exchange process - Deprotonation of $\mathrm{Me}_{2} \mathrm{NH}$ by an alkyl ligand-

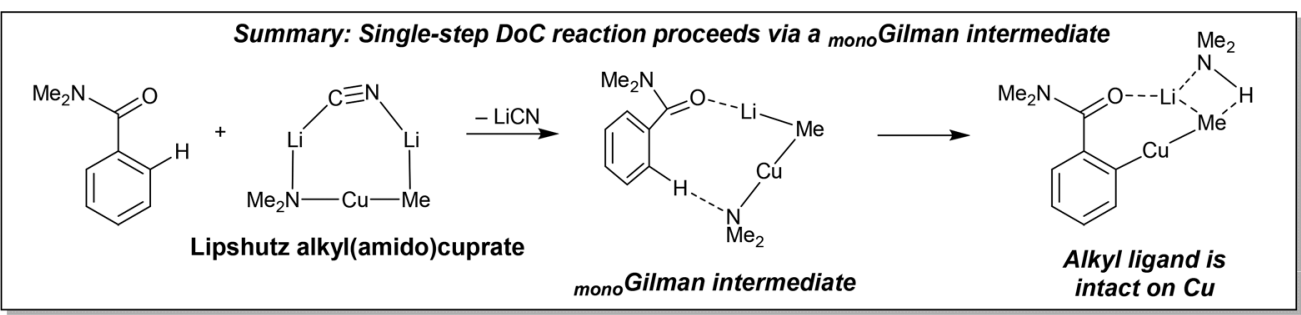

Scheme 14 Reaction pathways for the DoC of $N, N$-dimethylbenzamide using Lipshutz cuprate $M e C u\left(N M e_{2}\right)(C N) L_{2} \cdot 2 O M e_{2}$ and proceeding via a monoGilman intermediate. Energy changes and bond lengths at the B3LYP/6-31+G* and SVP $(\mathrm{Cu})$ level of theory are shown in $\mathrm{kcal}^{*} \mathrm{~mol}^{-1}$ and $\AA$, respectively.

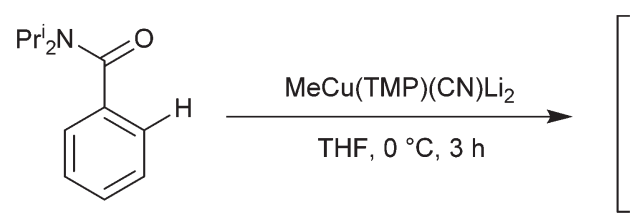<smiles>CCCNC(=O)c1ccccc1C(C)(C)N</smiles>

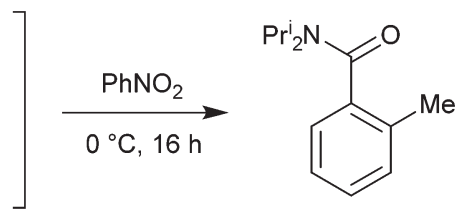

$93 \%$

Scheme 15 Oxidative ligand coupling employing an arylcuprate intermediate.

mide by $\mathrm{MeCu}(\mathrm{TMP})(\mathrm{CN}) \mathrm{Li}_{2}$ has been discussed in section 3.2 - with the use of nitrobenzene affording 2-methyl- $N, N$-diisopropylbenzamide (Scheme 15). ${ }^{6}$ This result clearly shows that the methyl ligand is retained by the copper centre in the arylcuprate intermediate and this bears out the theoretical work described here. A significant increase in the activation energy of deprotonation starting from a Gilman dimer has been found theoretically, ${ }^{32}$ again in agreement with experimental findings described in this section.
The most recent structural studies into bis(amido)cuprates have revealed the formation of triangulated complexes best viewed as adducts between Gilman and Lipshutz-type monomers. These are discussed in detail in section 2.4. Moreover, the adduct $\left[(\mathrm{DMP})_{2} \mathrm{CuLi} \cdot \mathrm{OEt}_{2}\right]_{2} \mathrm{LiBr} 29$ has been shown (see section 3.4) to be an effective DoC reagent. ${ }^{53}$ In the wake of recent DFT work, this has led to speculation that the adduct can deaggregate to give an active monoGilman base. In this vein, preliminary calculations on the simplified Lipshutz-type 

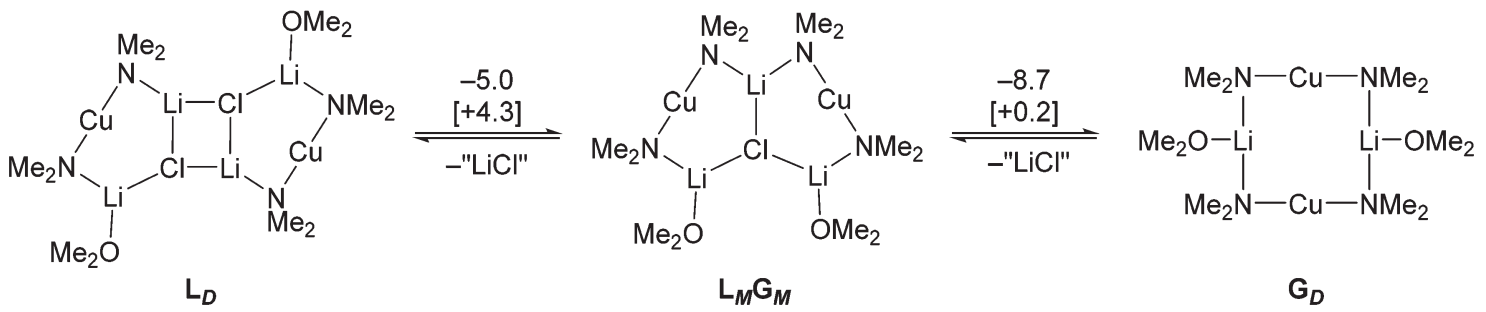

Scheme 16 The interconversion of a Lipshutz-type and a Gilman dimer $\left(L_{D}\right.$ and $\left.G_{D}\right)$ at B3LYP/631SVP level $\left(\Delta E[\Delta G]\right.$ are in kcal mol $\left.{ }^{-1}\right)$. "LiCl" $=$ $1 / 4\left[\mathrm{LiCl}\left(\mathrm{OMe}_{2}\right)\right]_{4}-\mathrm{OMe}_{2}$.

dimer of $\left(\mathrm{Me}_{2} \mathrm{~N}\right)_{2} \mathrm{Cu}(\mathrm{Cl}) \mathrm{Li}_{2} \cdot \mathrm{OMe}_{2}\left(\mathbf{L}_{\boldsymbol{D}}\right)$ have revealed an enthalpic preference for eliminating a solvated lithium halide to form Gilman dimer $\mathbf{G}_{\boldsymbol{D}}$ via adduct $\mathbf{L}_{M} \mathbf{G}_{M}$, though the effects of entropy mean that the system is a finely balanced one, as reflected by the very small values of $\Delta G$ (Scheme 16).

A further important step that it is necessary for $\mathbf{L}_{M} \mathbf{G}_{M}$ to participate in if it is to be viewed as a contender for DoC is its dissociation to generate $\mathbf{L}_{\boldsymbol{M}}$ and the participation of this in a pre-reaction complex with, for example, $N, N$-dimethylbenzamide. Data point to this sequence increasing $\Delta G$ by $6.1 \mathrm{kcal}$ mol $^{-1}$ with the initial dissociation of $\mathbf{L}_{M} \mathbf{G}_{M}$ raising $\Delta G$ by $15.4 \mathrm{kcal} \mathrm{mol}^{-1}$ (Scheme 17). In comparison to the $\mathbf{I M}_{G^{-}}$ TS2 $_{G}-\mathbf{I M} 4_{G}$ pathway modelled above (see Scheme 14) for sourcing monoGilman agents from a Lipshutz cuprate starting material, it is clear that the recently reported cuprate adducts can also be viewed as viable sources of monoGilman active species. This is consistent with their demonstrated ability (discussed in section 3.4) as DoC reagents. ${ }^{53}$

To summarize, DoM using Lipshutz-type amidocuprates or amidocuprate adducts can be viewed as proceeding via the formation of a mono Gilman intermediate, and in organyl(amido)cuprate systems the alkyl ligand is retained by the copper centre in the intermediate arylcuprate species, indicating that ligand scrambling does not occur.

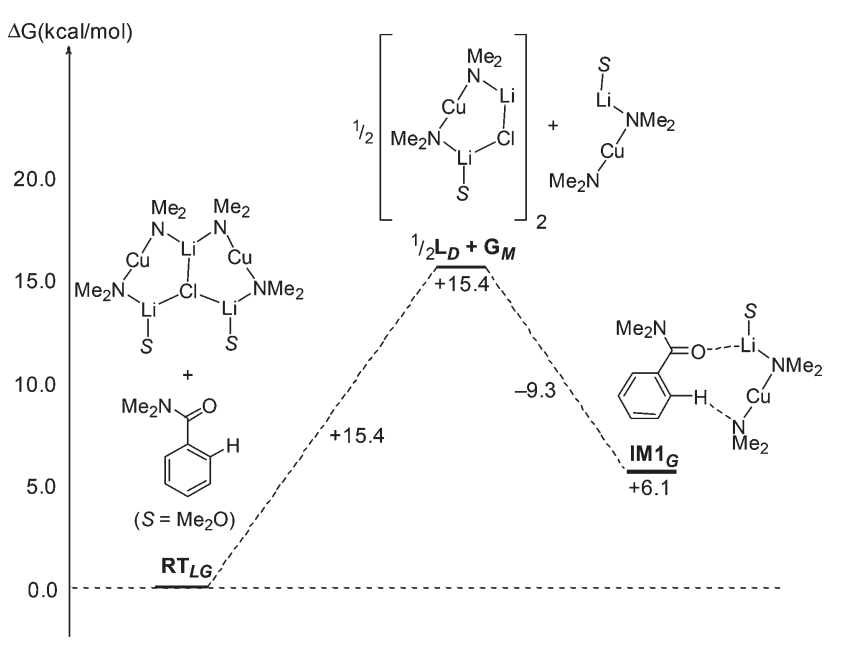

Scheme 17 Conversion of adduct $L_{M} G_{M}$ to a monoGilman-( $N, N$ dimethylbenzamide) complex $\left(\mathrm{IM} 1_{G}\right)$ at B3LYP/631SVP level.

\section{Conclusions}

The relatively new field of directed aromatic ortho metallation using synergic reagents has been significantly expanded through the deployment of lithium cuprates. Though these systems are not new in themselves it is only recently that unambiguous structural evidence for their nature has been forthcoming. Subsequent to spectroscopic and theoretical investigations on cyanide-containing lithium cuprates, it was only in 1998 that the first clear evidence for the behaviour of the cyanide ion was presented. ${ }^{23,24}$ More recently, the crystallographic elucidation of 7 revealed that the classic Lipshutz system $(\mathrm{TMP})_{2} \mathrm{Cu}(\mathrm{CN}) \mathrm{Li}_{2} \cdot \mathrm{THF}$ incorporated bonds between cyanide and lithium, with the lower polarity metal being ligated only by two diametrically opposed amide ligands. ${ }^{6}$ Gratifyingly, this observation was concordant with calculations reported as long ago as $1995 !^{61}$ Subsequent to this, it rapidly became apparent that the replacement of cyanide with halide $(=\mathrm{X})$ in $(\mathrm{TMP})_{2} \mathrm{Cu}(\mathrm{X}) \mathrm{Li}_{2} \cdot \mathrm{THF}(\mathrm{X}=\mathrm{Cl} \mathbf{9}, \mathrm{Br} \mathbf{1 0}, \mathrm{I} 12)$ had no very significant effect on lithium cuprate structure. This allows cyanide- and halide-containing TMP-cuprates to be discussed in terms of the existence of a series of cuprates that can be regarded as, to a greater or lesser extent, Lipshutz-type. This can be interpreted in terms of the elimination of LiX to give a Gilman cuprate (which is highly active, see below), though we have also seen that if $\mathrm{X}$ is very soft then unreactive Gilman dimers may be isolated. It should also be noted, though, that the observation of a common Lipshutz-type cuprate dimer for various $\mathrm{X}$ groups is also consistent with the recent suggestion that differing reactivities of cyanide and iodide complexes may be defined by the structures of reaction intermediates rather than those of the substrates themselves. ${ }^{7}$ That said, the general inefficiency of Gilman reagents in DoC is clear. The implication of these data is that for $\mathrm{D} o \mathrm{C}$ to proceed with optimum efficiency, a Lipshutz-type cuprate is necessary as the starting point.

Recent calculations have added a remarkable new insight to the structural and synthetic data noted above. Namely, that whereas the employment of a Lipshutz-type cuprate is necessary for efficient DoC to proceed (as evidenced synthetically and also proposed on the basis of reaction coordinates plotted in the previous section), the active species is a Gilman monomer created in situ by the expulsion of LiX from the Lipshutz-type substrate. DFT analysis reveals a compelling reaction pathway 
for the elimination of LiX prior to formation of a pre-reaction complex between aromatic substrate and Gilman monomer and, interestingly, also suggests that the formation of a reactive Gilman monomer from the corresponding dimer is, in fact, relatively unlikely. Again, this is borne out by structural and synthetic work.

Most recently, the isolation and characterisation of a new class of lithium cuprate that is best viewed as representing an adduct between Lipshutz-type and Gilman monomers has presented a new way of envisaging the in situ generation of reactive cuprates for DoC. The abstraction of LiX from a Lipshutztype dimer turns out to be enthalpically plausible. Moreover, calculations suggest that deaggregation of the resulting adduct and coordination of the formed (active) Gilman monomer by an aromatic substrate provides another compelling pathway for DoC. Overall, it is clear that, like Lipshutz-type dimers, the triangulated adducts reported here can be interpreted as plausible sources of the active cuprate species required for DoC.

The interpretation of theoretical data in terms of solid-state structures has afforded significant new insights into routes towards active monoGilman species. For example, adducts such as 28 have introduced new possibilities regarding the elimination of LiX from Lipshutz-type cuprates. Of course, these data have implications for our view of the solution behaviour of lithium cuprates and direct monitoring of the aggregation behaviour of these systems in solution, examples of which have been reported recently, ${ }^{42}$ will represent an increasingly significant part of forthcoming work. Attention is also turning to heteroleptic cuprates. Though these are reported for 19 and 20, heteroleptic bis(amido)cuprates represent the next target for structural elucidation and it is expected that they will be able to take two forms: the first will be mixed dimers or adducts such as $(\mathrm{AM})_{2} \mathrm{Cu}(\mathrm{X}) \mathrm{Li}_{2} \cdot\left(\mathrm{AM}^{\prime}\right)_{2} \mathrm{Cu}(\mathrm{X}) \mathrm{Li}_{2}$ and $(\mathrm{AM})_{2} \mathrm{Cu}(\mathrm{X})-$ $\mathrm{Li}_{2} \cdot\left(\mathrm{AM}^{\prime}\right)_{2} \mathrm{CuLi}$ while the second will take the form of structures that incorporate heteroleptic monomers such as $\mathrm{AMCu}$ $\left(\mathrm{AM}^{\prime}\right)(\mathrm{X}) \mathrm{Li}_{2}$ (where $\mathrm{AM}$ and $\mathrm{AM}^{\prime}$ are different amides). This work is rendered particularly important by the observation of distinctly contrasting amide orientations ( $c f$. TMP in $\mathbf{1 4}_{2}$ and DMP in 28) and attempts to fabricate mixed DMP/TMP-cuprates are ongoing. Moreover, the ability of DMP to result in relatively inexpensive cuprating agents opens up new possibilities in terms of varying the amide components of cuprate bases (e.g., different ring size, substitution pattern, introducing chirality) in order to develop a full understanding of the steric requirements necessary for effective DoC.

\section{Acknowledgements}

Much of this work was supported by the U.K. EPSRC (EP/ J500380/1). A.W. would like to acknowledge the graduate students who have contributed to the work in the past (Drs James Morey and Joanna Haywood) and also the GB Sasakawa and Daiwa Foundations and the Royal Society for support with travel and international collaboration. A.W. and M.U. thank the Japan Society for the Promotion of Science. M.U. and R.T. acknowledge Drs Shinsuke Komagawa, Shinya Usui, Ching-Yuan Liu, Yotaro Matsumoto and Keiichi Hirano, and Professors Yuichi Hashimoto, Shuji Yasuike and Jyoji Kurita for their contributions to the work. M.U. also thanks JSPS KAKENHI (S) (No. 24229011), Takeda Science Foundation, The Asahi Glass Foundation, Daiichi-Sankyo Foundation of Life Sciences, Mochida Memorial Foundation, Tokyo Biochemical Research Foundation, Foundation NAGASE Science Technology Development, Yamada Science Foundation and Sumitomo Foundation. R.T. acknowledges JSPS Grant-in-Aid for Young Scientists (A) (No. 25713001). F.C. and F.M. would like to acknowledge the graduate students who have contributed to the work (Dr Tan Tai Nguyen and Ms Nada Marquise) and the Agence Nationale de la Recherche (ACTIVATE program) for financial support. F.M. also thanks the Institut Universitaire de France and Rennes Métropole. Thanks go to Professor Paul Raithby and Dr Tom Robinson (Bath) for X-ray crystallographic studies. Calculations were performed on the RIKEN Super Combined Cluster (RSCC) and RIKEN Integrated Cluster of Clusters (RICC).

\section{References}

1 (a) H. W. Gschwend and H. R. Rodriguez, Org. React., 1979, 26, 1; (b) P. Beak and V. Snieckus, Acc. Chem. Res., 1982, 15, 306; (c) V. Snieckus, Chem. Rev., 1990, 90, 879; (d) T. G. Gant and A. I. Meyers, Tetrahedron, 1994, 50, 2297; (e) M. Schlosser, Organometallics in Synthesis, ed. M. Schlosser, Wiley, New York, 2nd edn, 2002, ch. 1.

2 Y. Kondo, M. Shilai, M. Uchiyama and T. Sakamoto, J. Am. Chem. Soc., 1999, 121, 3539.

3 (a) R. E. Mulvey, Organometallics, 2006, 25, 1060; (b) R. E. Mulvey, F. Mongin, M. Uchiyama and Y. Kondo, Angew. Chem., Int. Ed., 2007, 46, 3802; (c) R. E. Mulvey, Acc. Chem. Res., 2009, 42, 743; (d) B. Haag, M. Mosrin, H. Ila, V. Malakhov and P. Knochel, Angew. Chem., Int. Ed., 2011, 50, 9794; (e) F. Mongin and M. Uchiyama, Curr. Org. Chem., 2011, 15, 2340; $(f)$ R. E. Mulvey, Dalton Trans., 2013, 42, 6676; $(g)$ F. Mongin and A. Harrison-Marchand, Chem. Rev., 2013, 113, 7563.

4 (a) G. Wittig, Angew. Chem., 1958, 70, 65; (b) W. Tochtermann, Angew. Chem., Int. Ed. Engl., 1966, 5, 351.

5 A. Harrison-Marchand and F. Mongin, Chem. Rev., 2013, 113, 7470 .

6 S. Usui, Y. Hashimoto, J. V. Morey, A. E. H. Wheatley and M. Uchiyama, J. Am. Chem. Soc., 2007, 129, 15102.

7 M. Neumeier and R. M. Gschwind, J. Am. Chem. Soc., 2014, 136, 5765 .

8 H. Gilman, R. Jones and L. Woods, J. Org. Chem., 1952, 17, 1630.

9 H. House, W. Respess and G. Whitesides, J. Org. Chem., 1966, 31, 3128.

10 B. H. Lipshutz, R. S. Wilhelm and D. M. Floyd, J. Am. Chem. Soc., 1981, 103, 7672. 
11 G. Whitesides, W. Fisher, J. San Filipo, R. Bashe and H. House, J. Am. Chem. Soc., 1969, 91, 4871.

12 P. G. Edwards, R. W. Gellert, M. W. Marks and R. Bau, J. Am. Chem. Soc., 1982, 104, 2072.

13 H. Hope, D. Oram and P. P. Power, J. Am. Chem. Soc., 1984, 106, 1149.

14 (a) G. van Koten and J. G. Noltes, J. Chem. Soc., Chem. Commun., 1972, 940; (b) G. van Koten, J. T. B. H. Jastrzebski, F. Muller and C. H. Stam, J. Am. Chem. Soc., 1985, 107, 697.

15 G. van Koten and J. G. Noltes, J. Am. Chem. Soc., 1979, 101, 6593.

16 H. Hope, M. Olmstead, P. Power, J. Sandell and X. Xu, J. Am. Chem. Soc., 1985, 107, 4337.

17 H. O. House, D. G. Koepsell and W. J. Campbell, J. Org. Chem., 1972, 37, 1003.

18 S. Bertz, J. Am. Chem. Soc., 1990, 112, 4031.

19 B. H. Lipshutz, S. Sharma and E. L. Ellsworth, J. Am. Chem. Soc., 1990, 112, 4032.

20 J. Snyder, D. Spangler, J. Behling and B. Rossiter, J. Org. Chem., 1994, 59, 2665.

21 B. Lipshutz and B. James, J. Org. Chem., 1994, 59, 7585.

22 J. Snyder and S. Bertz, J. Org. Chem., 1995, 60, 4312.

23 C. Kronenberg, J. Jastrzebski, A. Spek and G. van Koten, J. Am. Chem. Soc., 1998, 120, 9688.

24 G. Boche, F. Bosold, M. Marsch and K. Harms, Angew. Chem., Int. Ed., 1998, 37, 1684.

25 D. R. Armstrong, D. Barr, R. Snaith, W. Clegg, R. E. Mulvey, K. Wade and D. Reed, J. Chem. Soc., Dalton Trans., 1987, 1071.

26 D. R. Armstrong, D. Barr, W. Clegg, S. M. Hodgeson, R. E. Mulvey, D. Reed, R. Snaith and D. S. Wright, J. Am. Chem. Soc., 1989, 111, 4719.

27 P. Reiss and D. Fenske, Z. Anorg. Allg. Chem., 2000, 626, 1317.

28 K. Snégaroff, T. T. Nguyen, N. Marquise, Y. S. Halauko, P. J. Harford, T. Roisnel, V. E. Matulis, O. A. Ivashkevich, F. Chevallier, A. E. H. Wheatley, P. C. Gros and F. Mongin, Chem. - Eur. J., 2011, 17, 13284.

29 N. Marquise, P. J. Harford, F. Chevallier, T. Roisnel, A. E. H. Wheatley, P. C. Gros and F. Mongin, Tetrahedron Lett., 2013, 54, 3154.

30 N. Marquise, P. J. Harford, F. Chevallier, T. Roisnel, V. Dorcet, A.-L. Gagez, S. Sablé, L. Picot, V. Thiéry, A. E. H. Wheatley, P. C. Gros and F. Mongin, Tetrahedron, 2013, 69, 10123.

31 C. M. P. Kronenburg, C. H. M. Amijs, J. T. B. H. Jastrzebski, M. Lutz, A. L. Spek and G. van Koten, Organometallics, 2002, 21, 4662.

32 S. Komagawa, S. Usui, J. Haywood, P. J. Harford, A. E. H. Wheatley, Y. Matsumoto, K. Hirano, R. Takita and M. Uchiyama, Angew. Chem., Int. Ed., 2012, 51, 12081.

33 R. P. Davies, Coord. Chem. Rev., 2011, 255, 1226.

34 J. Haywood, J. V. Morey, A. E. H. Wheatley, C.-Y. Liu, S. Yasuike, J. Kurita, M. Uchiyama and P. R. Raithby, Organometallics, 2009, 28, 38.
35 (a) S. Gambarotta, S. Strologo, C. Floriani and A. Chiesi-Villa, Organometallics, 1984, 3, 1444; (b) A. M. Dattelbaum and J. D. Martin, Polyhedron, 2006, 25, 349; (c) R. Fischer, H. Gorls and M. Westerhausen, Organometallics, 2007, 26, 3269.

36 (a) H. Hope, M. M. Olmstead, P. P. Power, J. Sandell and X. Xu, J. Am. Chem. Soc., 1985, 107, 4337; (b) R. P. Davies and S. Hornauer, Eur. J. Inorg. Chem., 2005, 51.

37 R. P. Davies and S. Hornauer, Chem. Commun., 2007, 304.

38 (a) M. Niemeyer, Organometallics, 1998, 17, 4649; (b) C.-S. Hwang and P. P. Power, Bull. Korean Chem. Soc., 2003, 24, 605.

39 (a) C.-S. Hwang and P. P. Power, J. Am. Chem. Soc., 1998, 120, 6409; (b) C.-S. Hwang and P. P. Power, J. Am. Chem. Soc., 1999, 18, 697.

40 R. Bomparola, R. P. Davies, T. Gray and A. J. P. White, Organometallics, 2009, 28, 4632.

41 R. M. Gschwind, Chem. Rev., 2008, 108, 3029.

42 R. P. Davies, S. Hornauer and P. B. Hitchcock, Angew. Chem., Int. Ed., 2007, 46, 5191.

43 (a) R. K. Dieter and M. Tokles, J. Am. Chem. Soc., 1987, 109, 2040; (b) R. K. Dieter and T. W. Hanks, Organometallics, 1992, 11, 3549; (c) B. E. Rossiter, M. Eguchi, G. Miao, N. M. Swingle, A. E. Hernandez, D. Vickers, E. Fluckiger, R. G. Patterson and K. V. Reddy, Tetrahedron, 1993, 49, 965. 44 M. John, C. Auel, C. Behrens, M. Marsch, K. Harms, F. Bosold, R. M. Gschwind, P. R. Rajamohanan and G. Boche, Chem. - Eur. J., 2000, 6, 3060.

45 S. F. Martin, J. R. Fishpaugh, J. M. Power, D. M. Giolando, R. A. Jones, C. M. Nunn and A. H. Cowley, J. Am. Chem. Soc., 1988, 110, 7226.

46 (a) M. Uchiyama, H. Naka, Y. Matsumoto and T. Ohwada, J. Am. Chem. Soc., 2004, 126, 10526; (b) H. Naka, M. Uchiyama, Y. Matsumoto, A. E. H. Wheatley, M. McPartlin, J. V. Morey and Y. Kondo, J. Am. Chem. Soc., 2007, 129, 1921; (c) J. García-Álvarez, E. Hevia, A. R. Kennedy, J. Klett and R. E. Mulvey, Chem. Commun., 2007, 2402; (d) H. Naka, J. V. Morey, J. Haywood, D. J. Eisler, M. McPartlin, F. García, H. Kudo, Y. Kondo, M. Uchiyama and A. E. H. Wheatley, J. Am. Chem. Soc., 2008, 130, 16193.

47 (a) M. Uchiyama, T. Miyoshi, Y. Kajihara, T. Sakamoto, Y. Otani, T. Ohwada and Y. Kondo, J. Am. Chem. Soc., 2002, 124, 8514; (b) W. Clegg, S. H. Dale, E. Hevia, G. W. Honeyman and R. E. Mulvey, Angew. Chem., Int. Ed., 2006, 45, 2370; (c) M. Uchiyama, Y. Matsumoto, D. Nobuto, T. Furuyama, K. Yamaguchi and K. Morokuma, J. Am. Chem. Soc., 2006, 128, 8748; (d) Y. Kondo, J. V. Morey, J. M. Morgan, P. R. Raithby, D. Nobuto, M. Uchiyama and A. E. H. Wheatley, J. Am. Chem. Soc., 2007, 129, 12734; (e) W. Clegg, J. Garcia-Álvarez, P. Garcia-Álvarez, D. V. Graham, R. W. Harrington, E. Hevia, A. R. Kennedy, R. E. Mulvey and L. Russo, Organometallics, 2008, 27, 2654. 48 J. Garcia-Álvarez, A. R. Kennedy, J. Klett and R. E. Mulvey, Angew. Chem., Int. Ed., 2007, 46, 1105. 
49 J. M. Cole, P. G. Waddell, A. E. H. Wheatley, G. J. McIntyre, A. J. Peel, C. W. Tate and D. J. Linton, Organometallics, 2014, DOI: 10.1021/om500271p.

50 P. P. Power, K. Ruhlandt-Senge and S. C. Shoner, Inorg. Chem., 1991, 30, 5013.

51 (a) R. Campbell, B. Conway, G. S. Fairweather, P. GarcíaÁlvarez, A. R. Kennedy, J. Klett, R. E. Mulvey, C. T. O’Hara and G. M. Robertson, Dalton Trans., 2010, 511; (b) B. Conway, P. García-Álvarez, A. R. Kennedy, J. Klett, R. E. Mulvey and S. D. Robertson, New J. Chem., 2010, 34, 1707.

52 D. R. Armstrong, J. A. Garden, A. R. Kennedy, S. A. Leenhouts, R. E. Mulvey, P. O'Keefe, C. T. O'Hara and A. Steven, Chem. - Eur. J., 2013, 19, 13492.

53 P. J. Harford, A. J. Peel, J. P. Taylor, S. Komagawa, P. R. Raithby, T. P. Robinson, M. Uchiyama and A. E. H. Wheatley, Chem. - Eur. J., 2014, 20, 3908.

54 C. M. P. Kronenburg, J. T. B. H. Jastrzebski, J. Boersma, M. Lutz, A. L. Spek and G. van Koten, J. Am. Chem. Soc., 2002, 124, 11675.

55 H.-J. Gais, C. V. Rao and R. Loo, Chem. - Eur. J., 2008, 14, 6510.

56 T. T. Nguyen, F. Chevallier, V. Jouikov and F. Mongin, Tetrahedron Lett., 2009, 50, 6787.
57 T. T. Nguyen, N. Marquise, F. Chevallier and F. Mongin, Chem. - Eur. J., 2011, 17, 10405.

58 (a) T. Fujisawa, Y. Kurita, M. Kawashima and T. Sato, Chem. Lett., 1982, 1641; (b) P. Kocienski and C. Barber, Pure Appl. Chem., 1990, 62, 1933; (c) P. Ashworth, B. Broadbelt, P. Jankowski, P. Kocienski, A. Pimm and R. Bell, Synthesis, 1995, 199.

59 (a) G. Queguiner, F. Marsais, V. Snieckus and J. Epsztajn, Adv. Heterocycl. Chem., 1991, 52, 187; (b) F. Mongin and G. Queguiner, Tetrahedron, 2001, 57, 4059; (c) A. Turck, N. Plé, F. Mongin and G. Queguiner, Tetrahedron, 2001, 57, 4489; (d) M. Schlosser and F. Mongin, Chem. Soc. Rev., 2007, 36, 1161; (e) F. Chevallier and F. Mongin, Chem. Soc. Rev., 2008, 37, 595; (f) P. C. Gros and Y. Fort, Eur. J. Org. Chem., 2009, 4199.

60 G. Queguiner, F. Marsais, V. Snieckus and J. Epsztajn, Adv. Heterocycl. Chem., 1991, 52, 187.

61 T. L. Stemmler, T. M. Barnhart, J. E. Penner-Hahn, C. E. Tucker, P. Knochel, M. Bohme and G. Frenking, J. Am. Chem. Soc., 1995, 117, 12489.

62 S. H. Bertz, G. Miao and M. Eriksson, Chem. Commun., 1996, 815. 